\title{
Mather problem and viscosity solutions in the stationary setting
}

\section{Diogo A. Gomes}

Center for Mathematical Analysis, Geometry, and Dynamical Systems

Departamento de Matemática, Instituto Superior Técnico 1049-001 Lisboa, Portugal

King Abdullah University of Science and Technology (KAUST), CSMSE Division

Thuwal 23955-6900. Saudi Arabia

E-mail address: dgomes@math.ist.utl.pt

\section{Elismar R. Oliveira}

Departamento de Matemática e Estatística

Universidade Federal do Rio Grande do Sul, Brazil

\begin{abstract}
In this paper we discuss the Mather problem for stationary Lagrangians, that is Lagrangians $L: \mathbb{R}^{n} \times \mathbb{R}^{n} \times \Omega \rightarrow \mathbb{R}$, where $\Omega$ is a compact metric space on which $\mathbb{R}^{n}$ acts through an action which leaves $L$ invariant. This setting allow us to generalize the standard Mather problem for quasi-periodic and almost-periodic Lagrangians. Our main result is the existence of stationary Mather measures invariant under the Euler-Lagrange flow which are supported in a graph. We also obtain several estimates for viscosity solutions of Hamilton-Jacobi equations for the discounted cost infinite horizon problem.
\end{abstract}

\section{Introduction}

Let $M$ be a complete compact manifold, and $L: T M \rightarrow \mathbb{R}$ a $C^{3}$ Lagrangian, fiberwise strictly convex and coercive. A probability measure on $T M$ is called holonomic if

$$
\int_{T M} v \cdot D \varphi d \mu=0
$$

for all $\varphi \in C^{1}(M)$. A central result in Aubry-Mather theory [Mn96] (see also [FS04]), is the fact that any holonomic probability measure $\mu$ on $T M$

D. Gomes was partially supported by CAMGSD-LARSys through FCTPortugal and by grants PTDC/MAT-CAL/0749/2012, UTA-CMU/MAT/0007/2009 PTDC/MAT/114397/2009, and UTAustin/MAT/0057/2008. 
which minimizes the action $\int_{T M} L d \mu$ is supported on a Lipschitz graph and is invariant under the Euler-Lagrange flow. Certain results in AubryMather theory have been extended for non-compact manifolds, see for instance [FM07, Mad06, or the very interesting paper [DS09 which addresses a problem related to ours in one dimension. However, as far as the authors know, there is in the literature no satisfactory construction of Mather measures for general non-compact manifolds.

In this paper, rather than considering Lagrangians on the tangent bundle of compact manifolds, such as in the original paper of Mather [Mat91], we consider Lagrangians defined on $\mathbb{R}^{n} \times \mathbb{R}^{n} \times \Omega$, where $\Omega$ is a suitable compact metric space on which $\mathbb{R}^{n}$ acts trough an action $\tau_{x}$. The main result of this paper is Theorem 16, in which we establish the existence of stationary Mather measures invariant under the Euler-Lagrange flow.

Stationary ergodic problems were considered in LS03 in the context of homogenization of random stationary ergodic Hamilton-Jacobi equations. The authors (in particular DG) are thankful to several enlightening discussions with P. Souganidis on this issue. Generalized Mather measures for stationary ergodic problems were also considered in the homogenization setting in GV07. The stationary ergodic setting was consider in DS09 where the construction of critical (or critical approximate) viscosity solutions of Hamilton-Jacobi equations is carried out in detail for the one-dimensional case. The stationary ergodic setting has been extensively studied in the context of homogenization of Hamilton-Jacobi equations, see [BDLLS08, LS05a, LS05b, Sou06, CSW05, Var08, KV08, KRV06.

A simple example (taken from LS03) which illustrates the main difficulties in the stationary setting is the Lagrangian

$$
L=\frac{|v|^{2}}{2}-\cos \left(x+\omega_{1}\right)-\cos \left(\sqrt{2} x+\omega_{2}\right) .
$$

Consider $\omega \in \mathbb{R}^{2} / \mathbb{Z}^{2} \equiv \mathbb{T}^{2}$ as a fixed parameter. It would be natural, as in Mather's problem, to look for probability measures $\mu$ on $\mathbb{R}^{n} \times \mathbb{R}^{n}$ which minimize the action

$$
\int_{\mathbb{R}^{n} \times \mathbb{R}^{n}} L d \mu
$$

under the holonomy constraint

$$
\int_{\mathbb{R}^{n} \times \mathbb{R}^{n}} v \cdot D_{x} \varphi d \mu=0,
$$

for all $\varphi$ of class $C^{1}$, bounded with bounded derivatives. This problem can be solved explicitly, and in fact we have the following two cases: if there exists a solution $\bar{x}$ to the overdetermined system

$$
\bar{x}+\omega_{1}=2 \pi m, \quad \sqrt{2} \bar{x}+\omega_{2}=2 \pi m,
$$

São Paulo J.Math.Sci. 6, 2 (2012), 301334 
for some $m \in \mathbb{Z}$, the Mather measure on $\mathbb{R} \times \mathbb{R}$ is simply $\mu_{0}=\delta_{\bar{x}}(x) \delta_{0}(v)$; otherwise there does not exist a Mather measure since $L>-1$ for all $(x, v)$, and the infimum in (1) is easily shown to be -1 .

To overcome these issues, which are due to the lack of compactness of $\mathbb{R}^{n}$, we will instead define stationary Mather measures as measures on $(v, \omega) \in \mathbb{R}^{n} \times \Omega$, which minimize the action and satisfy a suitable holonomy condition. It turns out that if $\Omega$ is compact and the Lagrangian satisfies certain stationarity hypothesis this is the natural way to generalize Mather measures. Before proceeding, we must make precise our framework.

Let $\Omega$ be a compact metric space, and let $L=L(x, v, \omega): \mathbb{R}^{n} \times \mathbb{R}^{n} \times$ $\Omega \rightarrow \mathbb{R}$ be a continuous Lagrangian, $C^{3}$ in the first two coordinates. The Lagrangian $L$ is also required to be strictly convex and superlinear on the velocity $v$, and nonnegative. In our setting, this last condition can be achieved without changing the nature of the problem by adding a constant to $L$. We assume further that

$$
L(x+y, v, \omega)-L(x, v, \omega) \leq|y|(C+C L(x, v, \omega)) .
$$

We suppose that there exists an action $\tau: \Omega \times \mathbb{R}^{n} \rightarrow \Omega$ which is continuous, satisfies the semigroup property

$$
\tau_{x+y} \omega=\tau_{x} \tau_{y} \omega \text { and } \tau_{0}(\cdot)=I d .
$$

Since $\Omega$ is compact and the action is continuous, the action is uniformly transitive $e^{1}$ in the following sense:

$$
\begin{aligned}
& \forall \varepsilon>0, \exists M>0, \forall \omega_{1}, \omega_{2} \in \Omega, \exists z \in \mathbb{R}^{n}, \\
& \text { such that }|z|<M, \text { and } d\left(\tau_{z} \omega_{1}, \omega_{2}\right)<\varepsilon .
\end{aligned}
$$

A first example of such an action is the following: we take $\Omega=\mathbb{T}^{d}$, the $d$-dimensional torus, let $n<d$ and we will construct an action $\tau$ : $\mathbb{R}^{n} \times \mathbb{T}^{d} \rightarrow \mathbb{T}^{d}$. To start with, we identify the torus $\mathbb{T}^{d}$ with its universal covering $\mathbb{R}^{d}$, and consider a constant coefficient $d \times n$ matrix $A$. Assume that $\left\{A x: x \in \mathbb{R}^{n}\right\}$ is dense in $\mathbb{T}^{d}$. Then we define

$$
\tau_{x} \omega=\omega+A x .
$$

A second example is the following. We take $\Omega$ to be the space of all sequences $\omega=\left(\omega_{k}\right)$ on $\mathbb{T}^{1}$, endowed with the following metric:

$$
d(\omega, \tilde{\omega})=\sum_{k=1}^{\infty} 2^{-k}\left|\omega_{k}-\tilde{\omega}_{k}\right| .
$$

\footnotetext{
${ }^{1}$ The authors are grateful to Albert Fathi that pointed out to us that uniform transitivity holds under the compactness assumption.
}

São Paulo J.Math.Sci. 6, 2 (2012), 301 334 
It is simple to verify that with this distance the space $\Omega$ is compact. A sequence $\lambda$ of real numbers is called irrational if for any $N$ the vector $\left(\lambda_{1}, \ldots, \lambda_{N}\right)$ is is linearly independent over the integers. Let $\bar{\lambda}$ be an irrational sequence. Define the following action from $\mathbb{R}$ into $\Omega$ by

$$
\tau_{x} \omega=\omega+x \bar{\lambda} .
$$

This action is also uniformly transitive.

$$
\begin{aligned}
& \text { A function } \varphi: \mathbb{R}^{n} \times \mathbb{R}^{n} \times \Omega \rightarrow \mathbb{R} \text {, is stationary if } \\
& \qquad \varphi(x+y, v, \omega)=\varphi\left(x, v, \tau_{y}(\omega)\right), \forall x, y \in \mathbb{R}^{n}, \omega \in \Omega .
\end{aligned}
$$

We assume that the Lagrangian $L$ is stationary.

Denote, in the strong topology,

$C_{s}^{1}\left(\mathbb{R}^{n} \times \Omega\right)=\left\{\varphi: \mathbb{R}^{n} \times \Omega \rightarrow \mathbb{R}\right.$, stationary, $C^{1}$ in the first variable, continuous in $\omega$, and such that $D_{x} \varphi(0, \omega)$ is continuous in $\left.\omega\right\}$, with an analogous definition for $C_{s}^{1}\left(\mathbb{R}^{n} \times \mathbb{R}^{n} \times \Omega\right)$.

If the action is given as in the first example by $\tau_{x} \omega=\omega+A x$, given $\psi$ : $\mathbb{T}^{d} \rightarrow \mathbb{R}$, the function $\varphi(x, \omega)=\psi(\omega+A x)$ is stationary, and, furthermore, $\varphi \in C_{s}^{1}$ if $\psi$ is $C^{1}$. In the second example we can construct an example of a stationary function in the following way: let $\psi_{k}: \mathbb{T} \rightarrow \mathbb{R}$ be a sequence of periodic functions uniformly bounded in $k$. Let

$$
\varphi(x, \omega)=\sum_{k} \psi_{k}\left(\omega_{k}+\bar{\lambda}_{k} x\right) 2^{-k} \frac{1}{1+\left|\bar{\lambda}_{k}\right|}
$$

Furthermore, if $\psi_{k}$ is $C^{1}$ and its derivatives are uniformly bounded in $k$, $\varphi \in C_{s}^{1}$.

To motivate the stationary Mather problem, let $x(t)$ be a globally Lipschitz trajectory on $\mathbb{R}^{n}$. Let $\omega_{0} \in \Omega$ be an arbitrary point. Consider ergodic averages to define an occupation measure $\mu$ on $\mathbb{R}^{n} \times \Omega$ corresponding to $x(\cdot)$ in the following way

$\lim _{T \rightarrow \infty} \frac{1}{T} \int_{0}^{T} \phi\left(x, \dot{x}, \omega_{0}\right) d t=\lim _{T \rightarrow \infty} \frac{1}{T} \int_{0}^{T} \phi\left(0, \dot{x}, \tau_{x} \omega_{0}\right) d t \equiv \int_{\mathbb{R}^{n} \times \Omega} \phi(0, v, \omega) d \mu$, where the limit is taken trough an appropriate sequence. Of course, the measure $\mu$ could depend on the point $\omega_{0}$ or the sequence through which the limit is taken. Nevertheless, such probabilities $\mu$, satisfy an integral constraint, the holonomy condition:

$$
\int_{\mathbb{R}^{n} \times \Omega} v \cdot D_{x} \varphi(0, \omega) d \mu=0,
$$

for any stationary function $\varphi \in C_{s}^{1}\left(\mathbb{R}^{n} \times \Omega\right)$.

São Paulo J.Math.Sci. 6, 2 (2012), 301334 
The stationary Mather problem can be formulated as follows: minimize

$$
\int_{\mathbb{R}^{n} \times \Omega} L(0, v, \omega) d \mu(v, \omega),
$$

over all probability measures that satisfy the holonomy constraint (3). A minimizing measure for this problem is called a stationary Mather measure. A similar problem arises also in [GV07] for the homogenization of HamiltonJacobi equations.

Let $\gamma: \mathbb{R}^{n} \rightarrow \mathbb{R}$ be a positive function such that

$$
\lim _{|v| \rightarrow \infty} \frac{|v|}{\gamma(v)}=0, \text { and } \lim _{|v| \rightarrow \infty} \frac{L(0, v, \omega)}{\gamma(v)}=+\infty
$$

where the last limit is uniform in $\omega \in \Omega$ by compactness. We denote by $C_{\gamma}^{0}\left(\mathbb{R}^{n} \times \Omega\right)$ the set of the continuous functions $\phi$ with

$$
\|\phi\|_{\gamma}=\sup _{\mathbb{R}^{n} \times \Omega} \frac{|\phi(v, \omega)|}{\gamma(v)}<\infty, \quad \lim _{|v| \rightarrow \infty} \frac{|\phi(v, \omega)|}{\gamma(v)} \rightarrow 0 .
$$

We will need also to consider the discounted Mather problem, see Gom08 for a discussion of related generalizations of Mather's problem. For that, let $\alpha$ be a positive number. Consider the operator $A^{v}: C_{s}^{1}\left(\mathbb{R}^{n} \times \Omega\right) \rightarrow$ $C_{\gamma}^{0}\left(\mathbb{R}^{n} \times \Omega\right)$ given by

$$
\varphi \rightarrow A^{v} \varphi(\omega)=v \cdot D_{x} \varphi(0, \omega)-\alpha \varphi(0, \omega) .
$$

The discounted stationary Mather problem consists in minimizing

$$
\int_{\mathbb{R}^{n} \times \Omega} L(0, v, \omega) d \mu(v, \omega)
$$

over all probability measures that satisfy the discounted holonomy constraint

$$
\int_{\mathbb{R}^{n} \times \Omega} A^{v} \varphi(\omega) d \mu(v, \omega)=-\alpha \int_{\Omega} \varphi(0, \omega) d \nu(\omega),
$$

for all $\varphi \in C_{s}^{1}\left(\mathbb{R}^{n} \times \Omega\right)$. A minimizing probability measure for this problem is called a discounted stationary Mather measure. The measure $\nu$ is called the trace of $\mu$. If $\alpha=0$ we call these measures stationary Mather measures.

The main result of this paper is the construction of stationary Mather measures invariant under the Euler-Lagrange flow. Usually, this flow is defined in $\mathbb{R}^{n} \times \mathbb{R}^{n}$. However, since the stationary Mather measures are measures on $\mathbb{R}^{n} \times \Omega$ we must now discuss the natural extension of the Euler-Lagrange flow to this space. 
Given a stationary vector field $W: \mathbb{R}^{n} \times \mathbb{R}^{n} \times \Omega \rightarrow \mathbb{R}^{n} \times \mathbb{R}^{n}$, let $\Phi=$ $\left(\Phi_{1}, \Phi_{2}\right): \mathbb{R} \times \mathbb{R}^{n} \times \mathbb{R}^{n} \times \Omega \rightarrow \mathbb{R}^{n} \times \mathbb{R}^{n}$ be its flow. We define the flow $\Psi: \mathbb{R} \times \mathbb{R}^{n} \times \Omega \rightarrow \mathbb{R}^{n} \times \Omega$ induced by $W$ in $\mathbb{R}^{n} \times \Omega$ as

$$
\Psi(t, v, \omega)=\left(\Phi_{2}(t, 0, v, \omega), \tau_{\Phi_{1}(t, 0, v, \omega)} \omega\right) .
$$

We denote by $C_{b}^{1}\left(\mathbb{R}^{n} \times \Omega\right)$ the set of bounded continuous functions $\phi(v, \omega)$ in $\mathbb{R}^{n} \times \Omega$ such that $D_{v} \phi(v, \omega)$ is also continuous and bounded. A measure $\mu$ is invariant under the flow $\Psi$ if,

$$
\int_{\mathbb{R}^{n} \times \Omega} \phi(\Psi(t, v, \omega)) d \mu(v, \omega)=\int_{\mathbb{R}^{n} \times \Omega} \phi(v, \omega) d \mu(v, \omega),
$$

for all $\phi \in C_{b}^{1}\left(\mathbb{R}^{n} \times \Omega\right)$ and for all $t \in \mathbb{R}$.

Let $\mu$ be a measure in $\mathbb{R}^{n} \times \Omega$ and $W: \mathbb{R}^{n} \times \mathbb{R}^{n} \times \Omega \rightarrow \mathbb{R}^{n} \times \mathbb{R}^{n}$ be a stationary vector field in $\mathbb{R}^{n} \times \mathbb{R}^{n}$. Then $\mu$ is invariant under the flow induced by $W$ in $\mathbb{R}^{n} \times \Omega$, if and only if,

$$
\int_{\mathbb{R}^{n} \times \Omega} \nabla \hat{\phi}(0, v, \omega) \cdot W(0, v, \omega) d \mu(v, \omega)=0,
$$

(where the gradient in the previous formula is taken both in $x$ and $v$ ) for all $\hat{\phi} \in \hat{C}_{\gamma}^{0}\left(\mathbb{R}^{n} \times \mathbb{R}^{n} \times \Omega\right)$. A proof for this classical fact for the case of vector fields on a manifold $M$ can be found, for instance, in [BG08]. The proof in our setting follows exactly along the same lines and we will omit it.

In this paper we will need to consider the discounted Lagrangian $L_{\alpha} \equiv$ $e^{-\alpha t} L(x, v)$. The corresponding Euler-Lagrange equation is

$$
\frac{d}{d t} D_{v} L(x, v, \omega)=D_{x} L(x, v, \omega)+\alpha D_{v} L,
$$

for each $\omega \in \Omega$. For $\alpha=0$ we obtain the usual Euler-Lagrange equations. We have a $\omega$-parametric Lagrangian vector field $W^{L_{\alpha}}$, that is given by:

$$
W^{L_{\alpha}}=\left\{\begin{array}{l}
X^{L_{\alpha}}(x, v, \omega)=v \\
Y^{L_{\alpha}}(x, v, \omega)=\left(D_{v v}^{2} L\right)^{-1}\left(D_{x} L+\alpha D_{v} L-D_{x v} L v\right) .
\end{array}\right.
$$

We say that a measure $\mu$ in $\mathbb{R}^{n} \times \Omega$ is invariant under the Euler-Lagrange flow if it is invariant under the flow $\Psi^{\alpha}$ induced by $W^{L_{\alpha}}$ in $\mathbb{R}^{n} \times \Omega$.

The outline of this paper is as follows: in section 2 we describe briefly the duality theory for the stationary Mather problem and its connections with viscosity solutions of Hamilton-Jacobi equations. Proofs of some the results, since they are standard, are outlined for completeness in appendix A. In section 3 we make some formal computations in the spirit of [EG01]. These computations suggest that for certain discounted stationary Mather measures one may be able to extend the regularity results in [EG01. Holonomic discounted stationary Mather measures are constructed in section 4 . 
Using these measures we obtain regularity results for viscosity solutions in section 5. These imply that the discounted stationary Mather measures are supported in a (partially) Lipschitz graph whose Lipschitz constant is independent of the discount factor $\alpha$. Finally in the last section we construct stationary Mather measures invariant under the Euler-Lagrange flow.

\section{Duality and viscosity solutions}

The stationary Mather problem is an infinite dimensional linear programming problem. As usual in these problems (see [Gom08, for instance), the duality theory plays an important role and will be developed in this section.

Theorem 1. Let $\nu$ be a probability measure on $\Omega$ and $\alpha \geq 0$. Define

$$
\bar{H}_{\alpha}=\inf \int_{\mathbb{R}^{n} \times \Omega} L(0, v, \omega) d \mu(v, \omega),
$$

where the infimum is taken over all probability measures on $\mathbb{R}^{n} \times \Omega$ which satisfy the discounted holonomy condition (5). Let

$$
\begin{aligned}
\mathcal{H}(\varphi, x, \omega) & =\sup _{v \in \mathbb{R}^{n}}\left(-A^{v}(\varphi)(x, \omega)-L(x, v, \omega)\right) \\
& =H\left(x, D_{x} \varphi(x, \omega), \omega\right)+\alpha \varphi(x, \omega),
\end{aligned}
$$

with $H(x, p, \omega)=\sup _{v \in \mathbb{R}^{n}}(-p \cdot v-L(x, v, \omega))$.

Then, the infimum in (8) is achieved at some probability measure $\mu$ satisfying (5) and furthermore

$$
\bar{H}_{\alpha}=-\inf _{\varphi \in C_{s}^{1}} \sup _{\Omega}\left\{-\alpha \int_{\Omega} \varphi d \nu+\mathcal{H}(\varphi, 0, \omega)\right\} .
$$

The proof of this Theorem is similar to analogous results in Gom08, for instance. For completeness, however, we present the proof in the Appendix A.

In this paper we will need to consider viscosity solutions to the equation

$$
\mathcal{H}^{\alpha}(u, 0, \omega) \equiv H\left(0, D_{x} u_{\alpha}(0, \omega), \omega\right)+\alpha u_{\alpha}(0, \omega)=0 .
$$

As in the standard Mather problem, viscosity solutions yield important information concerning the value of the variational problem (8), and help characterize the support of the measure.

Before we proceed, we make some remarks concerning the regularization by convolution of stationary functions.

Remark 1. To approximate a stationary function $u: \mathbb{R}^{n} \times \Omega \rightarrow \mathbb{R}$ by smooth stationary functions we are going to use a convolution with a standard mollifier $\eta^{\varepsilon}: \mathbb{R}^{n} \rightarrow \mathbb{R}$, that is, $\eta$ compactly supported, $\eta^{\varepsilon}(x)=\frac{1}{\varepsilon} \eta\left(\frac{x}{\varepsilon}\right)$, 
and $\int_{\mathbb{R}^{n}} \eta(x) d x=1$. We define the convolution between $u$ and $\eta^{\varepsilon}$ by

$$
u^{\varepsilon}(x, \omega)=\int_{\mathbb{R}^{n}} u\left(x, \tau_{y} \omega\right) \eta^{\varepsilon}(y) d y .
$$

Observe that, $u^{\varepsilon} \in C_{s}^{1}$. Moreover, we have

$$
\frac{\partial u^{\varepsilon}}{\partial x}(x, \omega) \cdot v=-\int_{\mathbb{R}^{n}} u\left(x, \tau_{y} \omega\right) D_{y} \eta^{\varepsilon}(y) \cdot v d y .
$$

We consider two different types of viscosity solutions for $\mathcal{H}(u, 0, \omega)=$ $\lambda$. Firstly recall the usual definition of viscosity solution: a function $u$ : $\mathbb{R}^{n} \times \Omega \rightarrow \mathbb{R}$, continuous in $x$ (not necessarily $C^{1}$ ) for each $\omega \in \Omega$, is a viscosity solution in $x$ of $\mathcal{H}(u, x, \omega)=\lambda$ if for each $\omega_{0} \in \Omega$, any $C^{1}$ function $\psi: \mathbb{R}^{n} \rightarrow \mathbb{R}$ and any $x_{0} \in \mathbb{R}^{n}$ such that $u\left(x, \omega_{0}\right)-\psi(x)$ has a strict local minimum (resp. maximum) at $x_{0}$ with $u\left(x_{0}, \omega_{0}\right)-\psi\left(x_{0}\right)=0$ we have

$$
\mathcal{H}\left(\psi, x_{0}, \omega_{0}\right) \geq \lambda(\text { resp. } \leq \lambda) .
$$

For our purposes we need a modified version of viscosity solution: a stationary (not necessarily $C^{1}$ ) function $u: \mathbb{R}^{n} \times \Omega \rightarrow \mathbb{R}$, continuous in $\Omega$, is a viscosity solution in $\omega$ of $\mathcal{H}(u, 0, \omega)=\lambda$ if for any $\varphi \in C_{s}^{1}\left(\mathbb{R}^{n} \times \Omega\right)$ and any point $\omega_{0} \in \Omega$ such that $u(0, \omega)-\varphi(0, \omega)$ has a local minimum (resp. maximum) at $\omega_{0}$ with $u\left(0, \omega_{0}\right)-\varphi\left(0, \omega_{0}\right)=0$ we have

$$
\mathcal{H}\left(\varphi, 0, \omega_{0}\right) \geq \lambda(\text { resp. } \leq \lambda)
$$

Proposition 2. Suppose that $u: \mathbb{R}^{n} \times \Omega \rightarrow \mathbb{R}$ is a viscosity solution in $x$ of $\mathcal{H}(u, 0, \omega)=\lambda$ and assume furthermore that $u$ is stationary and continuous in $\Omega$. Then $u$ is also a viscosity solution in $\omega$ of $\mathcal{H}(u, 0, \omega)=\lambda$.

Proof. Let $u: \mathbb{R}^{n} \times \Omega \rightarrow \mathbb{R}$ be a viscosity solution in $x$ of $\mathcal{H}(u, 0, \omega)=\lambda$. Consider an arbitrary function $\varphi \in C_{s}^{1}\left(\mathbb{R}^{n} \times \Omega\right)$ and a point $\omega_{0} \in \Omega$ such that $u(0, \omega)-\varphi(0, \omega)$ has a local minimum (resp. maximum) and $u\left(0, \omega_{0}\right)-$ $\varphi\left(0, \omega_{0}\right)=0$. Define $\psi(x)=\varphi\left(x, \omega_{0}\right)$. We claim that $u\left(x, \omega_{0}\right)-\psi(x)$ has a local minimum (resp. maximum) in $x_{0}=0 \in \mathbb{R}^{n}$. In fact,

$$
\begin{aligned}
u\left(x, \omega_{0}\right)-\psi(x) & =u\left(x, \omega_{0}\right)-\varphi\left(x, \omega_{0}\right)=u\left(0, \tau_{x} \omega_{0}\right)-\varphi\left(0, \tau_{x} \omega_{0}\right) \\
& \geq u\left(0, \omega_{0}\right)-\varphi\left(0, \omega_{0}\right)=u\left(0, \omega_{0}\right)-\psi(0), \quad(\text { resp. } \leq .)
\end{aligned}
$$

Then, because $u$ is a viscosity solution in $x$ we have

$$
\mathcal{H}\left(\psi, 0, \omega_{0}\right)=\mathcal{H}\left(\varphi, 0, \omega_{0}\right) \geq \lambda \quad(\text { resp. } \quad \leq \lambda)
$$


Consider the infinite horizon optimal control problem

$$
u_{\alpha}(x, \omega)=\inf _{x(0)=x} \int_{0}^{+\infty} e^{-\alpha t} L(x(t), \dot{x}(t), \omega) d t,
$$

where the infimum is taken over all globally Lipschitz trajectories with initial condition $x(0)=x$. Then $u_{\alpha}: \mathbb{R}^{n} \times \Omega \rightarrow \mathbb{R}$ satisfies the dynamic programing principle

$$
u_{\alpha}(x, \omega)=\inf _{x(0)=x}\left(\int_{0}^{T} e^{-\alpha t} L(x(t), \dot{x}(t), \omega) d t+e^{-\alpha T} u_{\alpha}(x(T), \omega)\right),
$$

among all globally Lipschitz trajectories with initial condition $x(0)=x$. It is standard, see [BCD97], that the function $u_{\alpha}$ is a viscosity solution of $\mathcal{H}(\varphi, 0, \omega)=0$ in $x$. Furthermore, the optimal trajectories are solutions to the discounted Euler-Lagrange equations (7). Finally, for $0<t<T$ we have additionally that $D_{x} u_{\alpha}(x(t))$ exists and

$$
\dot{x}(t)=-D_{p} H\left(D_{x} u_{\alpha}(x(t)), x(t)\right) .
$$

The next proposition is also a well known result, see, for instance, [BCD97] for similar results:

Proposition 3. For each $\omega$ fixed, let $u_{\alpha}(x, \omega)$ be a viscosity solution (in $x)$ of

$$
\mathcal{H}^{\alpha}(u, x, \omega)=H\left(x, D_{x} u_{\alpha}(x, \omega), \omega\right)+\alpha u_{\alpha}(x, \omega)=0 .
$$

Then $\alpha u_{\alpha}$ is uniformly bounded and $u_{\alpha}$ is uniformly Lipschitz in $x$, as $\alpha \rightarrow 0$.

Using standard techniques we can establish the following proposition, whose proof is presented in appendix B:

Proposition 4. Let $u_{\alpha}: \mathbb{R}^{n} \times \Omega \rightarrow \mathbb{R}$ be a solution of $(13)$. Then $u_{\alpha}$ is a viscosity solution (in $\omega$ ) of $\mathcal{H}(\varphi, 0, \omega)=0$, and $u_{\alpha}(0, \omega)$ is Lipschitz in $\omega$ with Lipschitz constant (in $\omega$ ) bounded by $K / \alpha$, where $K$ is independent of $\alpha$, for all $\alpha \geq 0$.

Proposition 5. Let $u_{\alpha}$ be a viscosity solution in $\omega$ of $(13)$ Then

$$
\inf _{\varphi \in C_{s}^{1}} \sup _{\omega \in \Omega}\left\{-\alpha \int_{\Omega} \varphi(0, \omega) d \nu(\omega)+\mathcal{H}^{\alpha}(\varphi, 0, \omega)\right\}=-\alpha \int_{\Omega} u_{\alpha}(0, \omega) d \nu(\omega) .
$$

Proof. Consider a viscosity solution $u_{\alpha}$ of $(13)$. Then for any $\varphi \in C_{s}^{1}$ there exists a point $\omega_{\varphi}$ of minimum for $u_{\alpha}(0, \omega)-\varphi(0, \omega)$. Consider $\varphi^{\prime}(x, \omega)=$ $\varphi(x, \omega)+\left(u_{\alpha}-\varphi\right)\left(0, \omega_{\varphi}\right)$. Then $u_{\alpha}(0, \omega)-\varphi^{\prime}(0, \omega)$ has a minimum equal to 0 in $\omega_{\varphi}$. 
Since $u_{\alpha}$ is a viscosity solution we have $\mathcal{H}^{\alpha}\left(\varphi^{\prime}, 0, \omega_{\varphi}\right) \geq 0$ or equivalently

$$
\mathcal{H}^{\alpha}\left(\varphi, 0, \omega_{\varphi}\right)+\alpha\left(u_{\alpha}-\varphi\right)\left(0, \omega_{\varphi}\right) \geq 0 \text {. }
$$

Therefore

$$
-\alpha \int_{\Omega} \varphi(0, \omega) d \nu+\mathcal{H}^{\alpha}\left(\varphi, 0, \omega_{\varphi}\right)+\alpha\left(u_{\alpha}-\varphi\right)\left(0, \omega_{\varphi}\right) \geq-\alpha \int_{\Omega} \varphi(0, \omega) d \nu
$$

which implies

$$
\sup _{\omega \in \Omega}-\alpha \int_{\Omega} \varphi(0, \omega) d \nu+\mathcal{H}^{\alpha}(\varphi, 0, \omega) \geq-\alpha \int_{\Omega} \varphi(0, \omega) d \nu+\alpha\left(u_{\alpha}-\varphi\right)\left(0, \omega_{\varphi}\right),
$$

and so

$$
\sup _{\omega \in \Omega}-\alpha \int_{\Omega} \varphi(0, \omega) d \nu+\mathcal{H}^{\alpha}(\varphi, 0, \omega) \geq-\alpha \int_{\Omega} u_{\alpha}(0, \omega) d \nu
$$

which finally yields

$$
\inf _{\varphi \in C_{s}^{1}} \sup _{\omega \in \Omega}\left\{-\alpha \int_{\Omega} \varphi(0, \omega) d \nu(\omega)+\mathcal{H}^{\alpha}(\varphi, 0, \omega)\right\} \geq-\alpha \int_{\Omega} u_{\alpha}(0, \omega) d \nu .
$$

In order to get the other inequality we use the functions $u^{\varepsilon}=u_{\alpha} * \eta_{\varepsilon}$. Then $\mathcal{H}^{\alpha}\left(u^{\varepsilon}, 0, \omega\right) \leq o(1)$ owing to the convexity of the Hamiltonian and the uniform Lipschitz estimates on $u_{\alpha}$, we have

$$
\inf _{\varphi \in C_{s}^{1}} \sup _{\omega \in \Omega}\left\{-\alpha \int_{\Omega} \varphi(0, \omega) d \nu(\omega)+\mathcal{H}^{\alpha}(\varphi, 0, \omega)\right\} \leq o(1)-\alpha \int_{\Omega} u^{\varepsilon}(0, \omega) d \nu .
$$

Then, the inequality desired is obtained by sending $\varepsilon$ to 0 , and ends the proof.

Corollary 6. We have

$$
\bar{H}_{\alpha}=\alpha \int_{\Omega} u_{\alpha}(0, \omega) d \nu
$$

where $u_{\alpha}$ is the unique viscosity solution of $H\left(0, D_{x} u_{\alpha}(0, \omega), \omega\right)+\alpha u_{\alpha}(0, \omega)=$ 0 .

Proof. In fact, if we apply Proposition 5 we have the formula

$$
\inf _{\varphi \in C_{s}^{1}} \sup _{\omega \in \Omega}\left\{-\alpha \int_{\Omega} \varphi(0, \omega) d \nu(\omega)+\mathcal{H}^{\alpha}(\varphi, 0, \omega)\right\}=-\alpha \int_{\Omega} u_{\alpha}(0, \omega) d \nu(\omega) .
$$

Remembering that $\bar{H}_{\alpha}=-\inf _{\varphi \in C_{s}^{1}} \sup _{\omega \in \Omega}-\alpha \int_{\Omega} \varphi(0, \omega) d \nu(\omega)+\mathcal{H}^{\alpha}(\varphi, 0, \omega)$, we get

$$
\bar{H}_{\alpha}=\alpha \int_{\Omega} u_{\alpha}(0, \omega) d \nu
$$

São Paulo J.Math.Sci. 6, 2 (2012), 301334 
We state next, without proof, a partial converse to Proposition 2, The proof is rather technical and, in this paper, its only application is in Remark 2 .

Proposition 7. Suppose that,

(a) There exists $\delta>0$ such that, for all $x \neq 0$ with $|x|<\delta, \tau_{x}(\cdot): \Omega \rightarrow \Omega$ does not have fixed points.

(b) For each $\omega_{0} \in \Omega$, there exists $\delta>0$ and a set $\Sigma_{\delta}\left(\omega_{0}\right) \ni \omega_{0}$, such that, for all $x \neq 0$ with $|x|<\delta$, and $\omega_{1}, \omega_{2} \in \Sigma_{\delta}\left(\omega_{0}\right)$, if $\tau_{x}\left(\omega_{1}\right)=\omega_{2}$ then $\omega_{1}=\omega_{2}$.

(c) The set

$$
\mathcal{U}_{\delta}\left(\omega_{0}\right)=\left\{\tau_{x}(\omega)\left|\omega \in \Sigma_{\delta}\left(\omega_{0}\right),\right| x \mid<\delta / 2\right\}
$$

is an open neighborhood of $\omega_{0}$.

If $u: \mathbb{R}^{n} \times \Omega \rightarrow \mathbb{R}$ is a viscosity solution in $\omega$ of $\mathcal{H}(u, 0, \omega)=\lambda$ then $u$ is also a viscosity solution in $x$ of $\mathcal{H}^{\alpha}(u, 0, \omega)=\lambda$.

Remark 2. Note that in some cases $\mathcal{H}^{\alpha}(u, \omega)=\lambda$ does not admit viscosity solutions in $\omega$, as pointed out in [LS03]. In their example $\Omega=\mathbb{T}^{2}, L=$ $L(x, v, \omega): \mathbb{R} \times \mathbb{R} \times \mathbb{T}^{2} \rightarrow \mathbb{R}$ is the Lagrangian given by $L(x, v, \omega)=\frac{1}{2} v^{2}+$ $\cos \left(\omega_{1}+x\right)+\cos \left(\omega_{2}+\sqrt{2} x\right)$, with the associated Hamiltonian $H(x, p, \omega)=$ $\frac{1}{2} p^{2}-\cos \left(\omega_{1}+x\right)-\cos \left(\omega_{2}+\sqrt{2} x\right)$, and the action $\tau: \mathbb{R} \times \mathbb{T}^{2} \rightarrow \mathbb{T}^{2}$ is given by $\tau_{x}\left(\omega_{1}, \omega_{2}\right)=\left(\omega_{1}+x, \omega_{2}+\sqrt{2} x\right)$.

In this case the viscosity solutions in $x$ are unbounded. So, if there where a viscosity solution in $\omega$, then it would be a solution in $x$ by Proposition 7. By compactness, any stationary continuous function is bounded, which would be a contradiction.

\section{Some formal computations}

In this section we adapt the formal computations in [EG01] to motivate the regularity results in the following sections. Consider the periodic case of a $C^{2}$ Lagrangian $L: \mathbb{T} \times \mathbb{R} \rightarrow \mathbb{R}$, given by $L(x, v)=\frac{1}{2} v^{2}-V(x)$, and the associated Hamiltonian $H(x, p)=\frac{1}{2} p^{2}+V(x)$. The stationary case follows along the same lines, as we will see in later sections.

Let $u$ be a solution to the discounted Hamilton-Jacobi equation $\frac{1}{2} u_{x}^{2}+$ $V(x)+\alpha u=0$. Let $\mu_{\alpha}$ be a discounted Mather measure with trace $\theta_{\alpha}$ and such that the projection of $\mu_{\alpha}$ in the $x$ coordinated is denoted by $\theta$, that is,

$$
\int_{\mathbb{T} \times \mathbb{R}} \varphi(x) d \mu_{\alpha}=\int_{\mathbb{T}} \varphi(x) d \theta .
$$


Note that $\theta$ in general does not agree with $\theta_{\alpha}$. In this section we assume that $\mu_{\alpha}$ has the special property that $\theta_{\alpha}=\theta$. Under this assumption $\mu_{\alpha}$ is holonomic, that is

$$
\int_{\mathbb{T} \times \mathbb{R}} v \varphi_{x}(x) d \mu_{\alpha}=0,
$$

for all $C^{1}$ periodic functions $\varphi(x)$.

We will first show that for $\mu_{\alpha}$ almost every $(x, v) \in \mathbb{T} \times \mathbb{R}$, we have $v=-u_{x}(x)$. To see this we will argue by contradiction. In this case if $v \neq-u_{x}(x)$, there would exist a set of positive measure $\mu_{\alpha}$ in which

$$
L(x, v)+v u_{x}>-H\left(u_{x}, x\right) .
$$

Since $L(x, v)+v u_{x} \geq-H\left(u_{x}, x\right)$, integrating with respect to $\mu_{\alpha}$ yields

$$
\int_{\mathbb{T} \times \mathbb{R}} L d \mu_{\alpha}+\int_{\mathbb{T} \times \mathbb{R}} v u_{x} d \mu_{\alpha}>\alpha \int_{\mathbb{T} \times \mathbb{R}} u d \mu_{\alpha} .
$$

This would yield

$$
\int_{\mathbb{T} \times \mathbb{R}} L d \mu_{\alpha}>\alpha \int_{\mathbb{T}} u d \theta_{\alpha},
$$

which contradicts the optimality condition.

Therefore the holonomy constraint can be written as

$$
\int_{\mathbb{R}}\left(u_{x} \varphi_{x}+\alpha \varphi\right) d \theta(x)=\alpha \int_{\mathbb{R}} \varphi d \theta_{\alpha}(x) .
$$

By differentiating twice the Hamilton-Jacobi equation we have $u_{x}\left(u_{x x}\right)_{x}+u_{x x}^{2}+V^{\prime \prime}(x)+\alpha u_{x x}=0$. Integrating with respect to $\mu_{\alpha}$ yields

$$
\int_{\mathbb{R}}\left(u_{x}\left(u_{x x}\right)_{x}+u_{x x}^{2}+V^{\prime \prime}(x)+\alpha u_{x x}\right) d \mu_{\alpha}=0,
$$

or, equivalently,

$$
\int_{\mathbb{R}} u_{x}\left(u_{x x}\right)_{x} d \theta(x)+\int_{\mathbb{R}} u_{x x}^{2}+\alpha u_{x x} d \theta(x)=-\int_{\mathbb{R}} V^{\prime \prime}(x) d \theta(x) .
$$

Since the trace of $\mu_{\alpha}, \theta_{\alpha}=\operatorname{tr} \mu_{\alpha}$, is equal to its projection $\theta(x)$, then the measure $\mu_{\alpha}$ is holonomic and so

$$
\int_{\mathbb{R}} u_{x}\left(u_{x x}\right)_{x} d \theta(x)=0 .
$$

São Paulo J.Math.Sci. 6, 2 (2012), 301334 
Using $-\alpha u_{x x} \leq \frac{1}{2} u_{x x}^{2}+\frac{1}{2} \alpha^{2}$ we get,

$$
\begin{aligned}
\int_{\mathbb{R}} u_{x x}^{2} d \theta(x) & =-\int_{\mathbb{R}} V^{\prime \prime}(x) d \theta(x)-\int_{\mathbb{R}} \alpha u_{x x} d \theta(x) \\
& \leq-\int_{\mathbb{R}} V^{\prime \prime}(x) d \theta(x)+\int_{\mathbb{R}} \frac{1}{2} u_{x x}^{2}+\frac{1}{2} \alpha^{2} d \theta(x),
\end{aligned}
$$

which yields a $L^{2}(\theta)$ bound for $u_{x x}$ :

$$
\int_{\mathbb{R}} u_{x x}^{2} d \theta(x) \leq \int_{\mathbb{R}} \alpha^{2}-2 V^{\prime \prime}(x) d \theta(x)
$$

In order to derive $L^{\infty}$ estimates to $u_{x x}$ we proceed as follows: first we multiply the second derivative of the Hamilton-Jacobi equation by a function $\Psi^{\prime}\left(u_{x x}\right)$,

$$
\int_{\mathbb{R}} u_{x}\left(u_{x x}\right)_{x} \Psi^{\prime}\left(u_{x x}\right) d \theta(x)+\int_{\mathbb{R}}\left[u_{x x}^{2}+\alpha u_{x x}+V^{\prime \prime}(x)\right] \Psi^{\prime}\left(u_{x x}\right) d \theta(x)=0 .
$$

Let $\Psi: \mathbb{R} \rightarrow \mathbb{R}$ be such that

$$
\Psi^{\prime}(x)= \begin{cases}1 & \text { if } x \leq-\lambda \\ 0 & \text { otherwise }\end{cases}
$$

where $\lambda>0$ is fixed. Choose $\Phi(x)=\Psi^{\prime}(x)$ (actually one should to use a $C^{\infty}$ approximation of $\left.\Psi^{\prime}(x)\right)$. Observe that $\left(u_{x x}\right)_{x} \Phi\left(u_{x x}\right)=\Psi\left(u_{x x}\right)_{x}$ and so $\int_{\mathbb{R}} u_{x}\left(u_{x x}\right)_{x} \Psi^{\prime}\left(u_{x x}\right) d \theta(x)=0$. Define $A=\left\{x \mid u_{x x} \leq-\lambda\right\}$. Thus,

$$
\begin{aligned}
0 & =\int_{\mathbb{R}}\left(u_{x x}^{2}+V^{\prime \prime}(x)+\alpha u_{x x}\right) \Phi\left(u_{x x}\right) d \theta(x) \\
& =\int_{A}\left(u_{x x}^{2}+V^{\prime \prime}(x)+\alpha u_{x x}\right) d \theta(x) .
\end{aligned}
$$

Since $u_{x x} \leq-\lambda$, and using $\alpha u_{x x} \leq-\frac{1}{2} u_{x x}^{2}-\frac{1}{2} \alpha^{2}$, one can show that, $0 \geq\left(\frac{\lambda^{2}}{2}-\frac{1}{2} \alpha^{2}+c\right) \theta(A)$, where, $\left|V^{\prime \prime}\right| \leq c$. Since $\lambda$ is arbitrary, we get $\theta(\bar{A})=0$. Thus, there exists $\lambda>0$, such that, $u_{x x}>-\lambda, \theta$-a.e.

The solutions of $\alpha u+\frac{1}{2} u_{x}^{2}+V(x)=0$ are semi-concave (this is a standard result, see [BCD97] or the survey paper [BG08]), so we get that there exists $\beta>0$ such that $u_{x x}<\beta$, and so, for some $C>0,\left|u_{x x}\right|<C, \theta$ almost everywhere.

\section{Holonomic discounted stationary Mather measures}

Motivated by the formal computations in the previous section, we will now establish the existence of holonomic discounted stationary Mather measures. In the paper [FCG08], these measures were called invariant, we did 
not keep this name here to avoid confusion with invariance with respect to Euler-Lagrange equations.

Given a probability measure $\nu$, and a corresponding discounted stationary Mather measure $\mu$ with trace $\nu$, we say that $\mu$ is a holonomic discounted stationary Mather measure if

$$
\int_{\mathbb{R}^{n} \times \Omega} \varphi(0, \omega) d \mu(v, \omega)=\int_{\Omega} \varphi(0, \omega) d \nu(\omega),
$$

for all $\varphi \in C_{s}^{1}\left(\mathbb{R}^{n} \times \Omega\right)$. In particular, $\mu$ satisfies the undiscounted holonomy constraint.

Theorem 8. There exists a holonomic discounted stationary Mather measure.

Proof. Fix $\omega \in \Omega$. Consider a sequence $T_{n} \rightarrow \infty$ and a sequence $x_{n}(t)$ of minimizing trajectories for the dynamic programing principle (12), that is,

$$
u_{\alpha}(0, \omega)=\int_{0}^{T_{n}} e^{-\alpha t} L\left(x_{n}(t), \dot{x}_{n}(t), \omega\right) d t+e^{-\alpha T_{n}} u_{\alpha}\left(x_{n}\left(T_{n}\right), \omega\right) .
$$

Because $u_{\alpha}$ is Lipschitz and

$$
\dot{x}_{n}=-D_{p} H\left(D_{x} u^{\alpha}\left(x_{n}(t)\right), x_{n}(t)\right)
$$

the $\left|\dot{x}_{n}\right|$ is uniformly bounded.

Define a probability measure $\mu$ by

$$
\int_{\mathbb{R}^{n} \times \Omega} \phi(v, \omega) d \mu(v, \omega)=\lim _{n \rightarrow \infty} \frac{1}{T_{n}} \int_{0}^{T_{n}} \phi\left(\dot{x}_{n}, \tau_{x_{n}(t)} \omega\right) d t
$$

for any $\phi \in C_{\gamma}^{0}\left(\mathbb{R}^{n} \times \Omega\right)$, where the limit is taken through an appropriate subsequence. This sublimit exists and is a probability measure because $\Omega$ is compact and $\left|\dot{x}_{n}\right|$ is uniformly bounded.

Let $\varphi \in C_{s}^{1}$. Observe that $\frac{d}{d t} \varphi\left(x_{n}(t), \omega\right)=\dot{x}_{n}(t) \cdot D_{x} \varphi\left(0, \tau_{x_{n}(t)} \omega\right)$. So, if $\phi(v, \omega)=v \cdot D_{x} \varphi(0, \omega)$, then

$$
\begin{aligned}
& \int_{\mathbb{R}^{n} \times \Omega} \phi(v, \omega) d \mu(v, \omega)=\lim _{n \rightarrow \infty} \frac{1}{T_{n}} \int_{0}^{T_{n}} \dot{x}_{n}(t) \cdot D_{x} \varphi\left(0, \tau_{x_{n}(t)} \omega\right) d t \\
& =\lim _{n \rightarrow \infty} \frac{1}{T_{n}} \int_{0}^{T_{n}} \frac{d}{d t} \varphi\left(x_{n}(t), \omega\right) d t=\lim _{n \rightarrow \infty} \frac{\varphi\left(x_{n}\left(T_{n}\right)\right)-\varphi\left(x_{n}(0)\right)}{T_{n}}=0 .
\end{aligned}
$$


Since $A^{v} \varphi=v \cdot D_{x} \varphi(0, \omega)-\alpha \varphi(0, \omega)$,

$$
\begin{aligned}
& \int_{\mathbb{R}^{n} \times \Omega} A^{v} \varphi d \mu=\int_{\mathbb{R}^{n} \times \Omega} v \cdot D_{x} \varphi(0, \omega)-\alpha \varphi(0, \omega) d \mu(v, \omega) \\
& =\lim _{n \rightarrow \infty} \frac{1}{T_{n}} \int_{0}^{T_{n}} \dot{x}_{n}(t) \cdot D_{x} \varphi\left(0, \tau_{x_{n}(t)} \omega\right)-\alpha \varphi\left(0, \tau_{x_{n}(t)} \omega\right) d t \\
& =-\alpha \lim _{T_{n} \rightarrow \infty} \frac{1}{T_{n}} \int_{0}^{T_{n}} \varphi\left(0, \tau_{x_{n}(t)} \omega\right) d t=-\alpha \int_{\Omega} \varphi(0, \omega) d \nu(\omega),
\end{aligned}
$$

where $\nu$ is given by,

$$
\int_{\Omega} g(\omega) d \nu(\omega)=\lim _{n \rightarrow \infty} \frac{1}{T_{n}} \int_{0}^{T_{n}} g\left(\tau_{x_{n}(t)} \omega\right) d t
$$

for all continuous function $g: \Omega \rightarrow \mathbb{R}$. In particular, $\int_{\mathbb{R}^{n} \times \Omega} \varphi(0, \omega) d \mu(v, \omega)=$ $\int_{\Omega} \varphi(0, \omega) d \nu(\omega)$.

We must prove that $\mu$ is minimizing. To do so, fix first $n$ and consider a partition $\left\{0=t_{0}, t_{1}, \ldots, t_{N-1}=T_{n}\right\}$ of $\left[0, T_{n}\right]$, where $t_{i+1}=t_{i}+h$, and $h=T_{n} / N$. The restriction of $x_{n}(t)$ to each sub-interval is minimizing, i.e.,

$$
u_{\alpha}\left(x_{n}\left(t_{i}\right), \omega\right)=\int_{t_{i}}^{t_{i+1}} e^{-\alpha\left(t-t_{i}\right)} L\left(x_{n}(t), \dot{x}_{n}(t), \omega\right) d t+e^{-\alpha h} u_{\alpha}\left(x_{n}\left(t_{i+1}\right), \omega\right) .
$$

We have,

$$
\begin{gathered}
\sum_{i=0}^{i=N-1} u_{\alpha}\left(x_{n}\left(t_{i}\right), \omega\right)-e^{-\alpha h} u_{\alpha}\left(x_{n}\left(t_{i+1}\right), \omega\right)= \\
=\sum_{i=0}^{i=N-1} u_{\alpha}\left(x_{n}\left(t_{i}\right), \omega\right)-u_{\alpha}\left(x_{n}\left(t_{i+1}\right), \omega\right)+\left(1-e^{-\alpha h}\right) u_{\alpha}\left(x_{n}\left(t_{i+1}\right), \omega\right)= \\
=u_{\alpha}\left(x_{n}(0), \omega\right)-u_{\alpha}\left(x_{n}(T), \omega\right)+\alpha\left(\frac{1-e^{-\alpha h}}{\alpha h}\right) \sum_{i=0}^{i=N-1} h u_{\alpha}\left(x_{n}\left(t_{i+1}\right), \omega\right) .
\end{gathered}
$$

Sending $h \rightarrow 0$ we get

$$
\begin{array}{r}
\lim _{h \rightarrow 0} \sum_{i=0}^{i=N-1} u_{\alpha}\left(x_{n}\left(t_{i}\right), \omega\right)-e^{-\alpha h} u_{\alpha}\left(x_{n}\left(t_{i+1}\right), \omega\right)= \\
u_{\alpha}\left(x_{n}(0), \omega\right)-u_{\alpha}\left(x_{n}\left(T_{n}\right), \omega\right)+\alpha \int_{0}^{T_{n}} u_{\alpha}\left(0, \tau_{x_{n}(t)} \omega\right) d t .
\end{array}
$$


On the other hand, we have

$$
\lim _{h \rightarrow 0} \sum_{i=0}^{i=N-1} \int_{t_{i}}^{t_{i+1}} e^{-\alpha\left(t-t_{i}\right)} L\left(x_{n}(t), \dot{x}_{n}(t), \omega\right) d t=\int_{0}^{T_{n}} L\left(x_{n}(t), \dot{x}_{n}(t), \omega\right) d t .
$$

Thus,

$$
\begin{aligned}
& \alpha \int u_{\alpha}(0, \omega) d \nu= \\
& \lim _{n \rightarrow \infty} \frac{1}{T_{n}}\left\{u_{\alpha}\left(x_{n}(0), \omega\right)-u_{\alpha}\left(x_{n}\left(T_{n}\right), \omega\right)+\alpha \int_{0}^{T_{n}} u_{\alpha}\left(0, \tau_{x_{n}(t)} \omega\right) d t\right\} \\
& =\lim _{n \rightarrow \infty} \frac{1}{T_{n}} \int_{0}^{T_{n}} L\left(x_{n}(t), \dot{x}_{n}(t), \omega\right) d t=\int_{\mathbb{R}^{n} \times \Omega} L(0, v, \omega) d \mu(v, \omega) .
\end{aligned}
$$

By Corollary 6 we have $\bar{H}_{\alpha}=\alpha \int u_{\alpha}(0, \omega) d \nu$. Thus $\mu$ is minimizing.

We should note here that the theorem does not assert uniqueness. Furthermore the measure $\mu$ may depend on the choice of $\omega \in \Omega$ or in the particular sequence we choose to extract the weak limits. For our purposes, however, existence is sufficient.

Theorem 9. Let $\mu_{\alpha}$ be a holonomic discounted Mather measure as constructed in Theorem 8. Then $\mu_{\alpha}$ is invariant under the discounted EulerLagrange flow.

Proof. It suffices to prove that for any bounded function $\phi(x, v, \omega) \in C_{s}^{1}\left(\mathbb{R}^{n} \times\right.$ $\left.\mathbb{R}^{n} \times \omega\right)$ we have

$$
\int_{\mathbb{R}^{n} \times \Omega} W^{L_{\alpha}} \nabla_{x, v} \phi(0, v, \omega) d \mu_{\alpha}=0 .
$$

This follows, from the identity

$$
\begin{aligned}
\phi\left(x_{n}\left(T_{n}\right), \dot{x}_{n}\left(T_{n}\right), \omega\right)-\phi\left(x_{n}(0), \dot{x}_{n}(0), \omega\right) & =\int_{0}^{T_{n}} \frac{d}{d t} \phi\left(x_{n}(t), \dot{z}_{n}(t), \omega\right) \\
& =\int_{0}^{T_{n}} X^{L_{\alpha}} \frac{\partial \phi}{\partial x}+Y^{L_{\alpha}} \frac{\partial \phi}{\partial v}
\end{aligned}
$$

dividing by $T_{n}$ and letting $n \rightarrow \infty$.

\section{Graph property, regularity and stationary Mather mea- sures}

In this section we establish that the discounted Mather measures are supported in a graph of a (partially) Lipschitz function. As we are using similar techniques to [EG01] (see also [BG08]) we will present in this section 
the main differences and technical points and postpone to Appendix C the detailed proofs. We will use the discounted Mather measures to construct a stationary Mather measure invariant under the Euler-Lagrange flow.

We assume that

$$
L(x+y, v, \omega)-L(x, v, \omega) \leq(c+c L)|y|
$$

Lemma 10. Let $u_{\alpha}$ be the viscosity solution of $H\left(0, D_{x} u_{\alpha}(0, \omega), \omega\right)+$ $\alpha u_{\alpha}(0, \omega)=0$ given by Proposition 4 . Then

$$
\lim _{\alpha \rightarrow 0} \alpha u_{\alpha}(0, \omega)
$$

does not depend on $\omega$.

Proof. We know that $\alpha u_{\alpha}$ is uniformly bounded, so $\alpha u_{\alpha}(0, \omega) \rightarrow \xi(\omega)$ pointwise for some function. On the other hand, fixed $\omega_{0} \in \Omega$ we know that $u_{\alpha}\left(y, \omega_{0}\right)$ is uniformly Lipschitz in $x$, uniformly as $\alpha \rightarrow 0$, that is,

$$
\left|u_{\alpha}\left(x_{1}, \omega_{0}\right)-u_{\alpha}\left(x_{2}, \omega_{0}\right)\right|<C\left|x_{1}-x_{2}\right| .
$$

Thus, if $|y|<R$ then,

$$
\lim _{\alpha \rightarrow 0}\left|\alpha u_{\alpha}\left(y, \omega_{0}\right)-\alpha u_{\alpha}\left(0, \omega_{0}\right)\right|<\lim _{\alpha \rightarrow 0} \alpha C|y|=0,
$$

that is, $\lim _{\alpha \rightarrow 0} \alpha u_{\alpha}\left(0, \tau_{y} \omega_{0}\right)=\lim _{\alpha \rightarrow 0} \alpha u_{\alpha}\left(0, \omega_{0}\right)$ for $|y|<R$.

From Proposition 4 we know that $u_{\alpha}(0, \omega)$ is Lipschitz in $\omega$ with Lipschitz constant $K / \alpha$, that is,

$$
\left|u_{\alpha}\left(0, \omega_{1}\right)-u_{\alpha}\left(0, \omega_{2}\right)\right|<\frac{K}{\alpha} d\left(\omega_{1}, \omega_{2}\right) .
$$

Consider $\varepsilon>0$ and $y \in \mathbb{R}^{n}$, such that $d\left(\tau_{y} \omega_{0}, \omega_{1}\right)<\varepsilon$. Observe that,

$$
\begin{gathered}
\left|\alpha u_{\alpha}\left(0, \omega_{0}\right)-\alpha u_{\alpha}\left(0, \omega_{1}\right)\right| \leq \\
\leq\left|\alpha u_{\alpha}\left(0, \omega_{0}\right)-\alpha u_{\alpha}\left(0, \tau_{y} \omega_{0}\right)\right|+\left|\alpha u_{\alpha}\left(0, \tau_{y} \omega_{0}\right)-\alpha u_{\alpha}\left(0, \omega_{1}\right)\right| \leq \\
\leq\left|\alpha u_{\alpha}\left(0, \omega_{0}\right)-\alpha u_{\alpha}\left(0, \tau_{y} \omega_{0}\right)\right|+\alpha \frac{K}{\alpha} d\left(\tau_{y} \omega_{0}, \omega_{1}\right) .
\end{gathered}
$$

Sending $\alpha \rightarrow 0$, and then $\varepsilon \rightarrow 0$ we get $\lim _{\alpha \rightarrow 0} \alpha u_{\alpha}\left(0, \omega_{0}\right)=\lim _{\alpha \rightarrow 0} \alpha u_{\alpha}\left(0, \omega_{1}\right)$. Thus, $\xi(\omega)$ is constant.

Lemma 11. Let $u_{\alpha}$ be the viscosity solution of $H\left(0, D_{x} u_{\alpha}(0, \omega), \omega\right)+$ $\alpha u_{\alpha}(0, \omega)=0$ given by Proposition 4. Then

$$
\lim _{\alpha \rightarrow 0} \alpha u_{\alpha}(0, \omega)=\bar{H}
$$

where

$$
\bar{H}=\inf \int_{\mathbb{R}^{n} \times \Omega} L d \mu,
$$

and the infimum is taken over all stationary holonomic measures. 
Proof. Denote by $\tilde{H}$ the limit as $\alpha \rightarrow 0$ of $\alpha u_{\alpha}$, which is constant by the previous lemma. Let $\mu_{\alpha}$ be a holonomic discounted stationary Mather measure. Then, because $\mu_{\alpha}$ is holonomic we have

$$
\bar{H} \leq \lim _{\alpha \rightarrow 0} \int_{\mathbb{R}^{n} \times \Omega} L d \mu_{\alpha}=\lim _{\alpha \rightarrow 0} \alpha \int_{\mathbb{R}^{n} \times \Omega} u_{\alpha} d \mu_{\alpha}=\tilde{H} .
$$

Let $\mu$ be a stationary Mather measure. Then, because $\mu$ is a discounted holonomic measure with trace $\mu$ we have

$$
\bar{H}=\int_{\mathbb{R}^{n} \times \Omega} L d \mu \geq \alpha \int_{\mathbb{R}^{n} \times \Omega} u_{\alpha} d \mu \rightarrow \tilde{H},
$$

as $\alpha \rightarrow 0$. This shows that $\tilde{H}=\bar{H}$.

Lemma 12. Let $\mu_{\alpha}$ be a sequence of discounted stationary Mather measures with trace $\nu_{\alpha}$. Suppose that $\mu_{\alpha} \rightarrow \mu$ when $\alpha \rightarrow 0$, then $\mu$ is a stationary Mather measure.

Proof. First we must prove that $\mu$ is a holonomic probability measure. In fact, for any $\varphi \in C_{s}^{1}$,

$$
\int_{\mathbb{R}^{n} \times \Omega} v \cdot D_{x} \varphi(0, \omega) d \mu_{\alpha}=\alpha \int_{\mathbb{R}^{n} \times \Omega} \varphi(0, \omega) d \mu_{\alpha}-\alpha \int_{\Omega} \varphi(0, \omega) d \nu_{\alpha} \rightarrow 0,
$$

when $\alpha \rightarrow 0$.

Using Corollary 6 we get

$$
\int_{\mathbb{R}^{n} \times \Omega} L d \mu=\lim _{\alpha \rightarrow 0} \int_{\mathbb{R}^{n} \times \Omega} L d \mu_{\alpha}=\lim _{\alpha \rightarrow 0} \bar{H}_{\alpha}=\lim _{\alpha \rightarrow 0} \int_{\Omega} \alpha u_{\alpha}(0, \omega) d \nu_{\alpha}(\omega)=\bar{H} .
$$

Thus $\mu$ is a Mather measure.

Theorem 13. Let $\mu_{\alpha}$ be a discounted Mather measure with trace $\nu_{\alpha}$ (or if $\alpha=0$ a stationary Mather measure). Then $\mu_{\alpha}$ is supported in a graph, that is, there exists a measurable function $V_{\alpha}: \Omega \rightarrow \mathbb{R}^{n}$ such that,

$$
\operatorname{supp} \mu_{\alpha}=\left\{(v, \omega) \in \mathbb{R}^{n} \times \Omega \mid v=V_{\alpha}(\omega)\right\} .
$$

Proof. As in [BG08, for instance, we just observe that the result follows from the fact that the Lagrangian is strictly convex in $v$, whereas the discounted holonomy constraint is linear in $v$.

Since the holonomic discounted measures are also holonomic, the same techniques in [EG01] (see also [BG08]) can be adapted to establish the following regularity result:

São Paulo J.Math.Sci. 6, 2 (2012), 301334 
Theorem 14. Let $\mu_{\alpha}$ be a holonomic discounted Mather measure. If $u_{\alpha}$ is a viscosity solution of $(10)$, then for each $y \in \mathbb{R}$,

$$
\left|D_{x} u_{\alpha}(y, \omega)-D_{x} u_{\alpha}(0, \omega)\right| \leq C|y|,
$$

$\theta$ almost everywhere and uniformly in $\alpha$.

The proof of this theorem follows (almost) exactly the same steps as in [EG01] (see also [BG08]) and is presented for completeness in appendix C. The only difference is the term $\alpha u$ in the Hamilton-Jacobi equation, which can be controlled, as discussed in section 3 , because we are using holonomic discounted measures. As a corollary to the previous theorem we have

Corollary 15. Let $\mu_{\alpha}$ be a holonomic discounted Mather measure. Then, there exists a function $V_{\alpha}: \Omega \rightarrow \mathbb{R}^{n}$, such that $\operatorname{supp} \mu_{\alpha}=\left\{(v, \omega) \in \mathbb{R}^{n} \times\right.$ $\left.\Omega \mid v=V_{\alpha}(\omega)\right\}$. Furthermore, $V_{\alpha}$ is partially Lipschitz in the following sense:

$$
\left|V_{\alpha}\left(\tau_{y} \omega\right)-V_{\alpha}(\omega)\right| \leq C|y|
$$

for all $\omega$ in the support of $\mu_{\alpha}$, and $C$ is uniformly bounded as $\alpha \rightarrow 0$.

Finally, our last result concerns the existence of stationary Mather measures invariant under the Euler-Lagrange flow.

Theorem 16. There exists a stationary Mather measure $\mu$ which is invariant under the Euler-Lagrange flow. Furthermore $\mu$ is supported on a graph.

Proof. Let $\mu_{\alpha}$ be holonomic discounted Mather measures as constructed in Theorem 8. Consider a weak limit $\mu$. By Lemma 12, $\mu$ is a stationary Mather measure. Because for any $\phi(x, v)$ we have

$$
\int_{\mathbb{R}^{n} \times \Omega} W^{L_{0}} \nabla_{x, v} \phi(0, v, \omega) d \mu_{\alpha}=\alpha \int_{\mathbb{R}^{n} \times \Omega}\left(D_{v v}^{2} L\right)^{-1} D_{v} L D_{v} \phi(0, v, \omega) d \mu_{\alpha} .
$$

we conclude that

$$
\int_{\mathbb{R}^{n} \times \Omega} W^{L_{0}} \nabla_{x, v} \phi(0, v, \omega) d \mu=0 .
$$

The graph property of stationary Mather measures follows from Theorem 13 .

\section{A. Proof of Theorem 1}

In this appendix we present the proof of Theorem 1, as well as some background material. 
Let $\gamma$ be as in (4). Let $\mathcal{M}$ be the set of weighted Radon measures on $\Omega \times \mathbb{R}^{n}$, i.e.,

$$
\mathcal{M}=\left\{\text { signed measures on } \mathbb{R}^{n} \times \Omega \text { with } \int_{\mathbb{R}^{n} \times \Omega} \gamma d|\mu|<\infty\right\} .
$$

Note that $\mathcal{M}$ is the dual of the set $C_{\gamma}^{0}\left(\mathbb{R}^{n} \times \Omega\right)$.

We introduce the following sets

$$
\mathcal{M}_{1}=\{\mu \in \mathcal{M} \mid \mu \text { is a positive probability measure }\},
$$

and

$$
\mathcal{M}_{2}=\left\{\mu \in \mathcal{M} \mid \int_{\mathbb{R}^{n} \times \Omega} v \cdot D_{x} \varphi(0, \omega) d \mu(v, \omega)=0, \text { for all } \varphi \in C_{s}^{1}\left(\mathbb{R}^{n} \times \Omega\right)\right\} .
$$

Using this notation the Mather problem can be reformulated as

$$
\min _{\mathcal{M}_{1} \cap \mathcal{M}_{2}} \int_{\mathbb{R}^{n} \times \Omega} L(0, v, \omega) d \mu(v, \omega) .
$$

Consider the following subset of functions $\phi: \mathbb{R}^{n} \times \Omega \rightarrow \mathbb{R}$,

$$
\mathcal{C}=\operatorname{cl}\left\{\phi \mid \phi(v, \omega)=v \cdot D_{x} \varphi(0, \omega), \text { for some } \varphi \in C_{s}^{1}\left(\mathbb{R}^{n} \times \Omega\right)\right\} .
$$

Observe that $\mathcal{C}$ is a closed convex set.

For $\phi \in C_{\gamma}^{0}$ let

$$
h(\phi)=\sup _{\mathbb{R}^{n} \times \Omega}(-\phi(v, \omega)-L(0, v, \omega)) .
$$

Since $h$ is the supremum of linear functions, it is a convex function on $C_{\gamma}^{0}$. As we will see bellow in Lemma $18, h$ is a continuous function.

For $\phi \in C_{\gamma}^{0}$, let

$$
g(\phi)= \begin{cases}0 & \text { if } \phi \in \mathcal{C} \\ -\infty & \text { otherwise }\end{cases}
$$

As $\mathcal{C}$ is a closed convex set we have that $g$ is a concave and upper semicontinuous function. Therefore its Legendre-Fenchel transform is given by

$$
g^{*}(\mu)=\inf _{\phi \in C_{\gamma}^{0}\left(\mathbb{R}^{n} \times \Omega\right)}\left(-\int_{\mathbb{R}^{n} \times \Omega} \phi d \mu-g(\phi)\right) .
$$

Since $h$ is a convex and lower semicontinuous function, its Legendre-Fenchel transform is given by

$$
h^{*}(\mu)=\sup _{\phi \in C_{\gamma}^{0}\left(\mathbb{R}^{n} \times \Omega\right)}\left(-\int_{\mathbb{R}^{n} \times \Omega} \phi d \mu-h(\phi)\right) .
$$


Proposition 17. Let $g$ and $h$ defined as in (15) and (16). Then

$$
h^{*}(\mu)= \begin{cases}\int_{\mathbb{R}^{n} \times \Omega} L(0, v, \omega) d \mu(v, \omega) & \text { if } \mu \in \mathcal{M}_{1} \\ +\infty & \text { otherwise }\end{cases}
$$

and

$$
g^{*}(\mu)= \begin{cases}0 & \text { if } \mu \in \mathcal{M}_{2} \\ -\infty & \text { otherwise }\end{cases}
$$

Proof. First we assume that $\mu \in \mathcal{M}_{1}$. As $h$ is a convex function, its Legendre transform is given by (18). Using the definition of $h$, equation (15), we get

$$
h^{*}(\mu)=\sup _{\phi \in C_{\gamma}^{0}\left(\mathbb{R}^{n} \times \Omega\right)}\left(-\int_{\mathbb{R}^{n} \times \Omega} \phi d \mu-\sup _{\mathbb{R}^{n} \times \Omega}(-\phi(v, \omega)-L(0, v, \omega))\right) .
$$

Consider the family of compact subsets of $\mathbb{R}^{n} \times \Omega$ given by

$$
K_{n}=\left\{(v, \omega) \in \mathbb{R}^{n} \times \Omega|| v \mid \leq n\right\},
$$

and let $\eta_{n}: \mathbb{R}^{n} \times \Omega \rightarrow \mathbb{R}$ be a continuous function such that $0 \leq \eta_{n} \leq 1$, $\eta_{n}=1$ in $K_{n}$, and $\operatorname{supp} \eta_{n} \subset K_{n+1}$. Then define

$$
L_{n}=L(0, v, \omega) \cdot \eta_{n}(v, \omega) .
$$

Observe that the sequence $L_{n}$ is increasing and pointwise convergent to $L(0, v, \omega)$.

Is easy to see that $L_{n} \in C_{\gamma}^{0}\left(\mathbb{R}^{n} \times \Omega\right)$. Furthermore, for fixed $n$, one can write any function $\phi \in C_{\gamma}^{0}\left(\mathbb{R}^{n} \times \Omega\right)$ as $\phi=-L_{n}+\psi$ where $\psi \in C_{\gamma}^{0}\left(\mathbb{R}^{n} \times \Omega\right)$. From this observation we get

$$
\begin{aligned}
h^{*}(\mu) & =\sup _{\psi \in C_{\gamma}^{0}\left(\mathbb{R}^{n} \times \Omega\right)}\left(-\int_{\mathbb{R}^{n} \times \Omega}\left(-L_{n}+\psi\right) d \mu-\sup _{\mathbb{R}^{n} \times \Omega}\left(-\left(-L_{n}+\psi\right)-L\right)\right) \\
& =\sup _{\psi \in C_{\gamma}^{0}\left(\mathbb{R}^{n} \times \Omega\right)}\left(\int_{\mathbb{R}^{n} \times \Omega} L_{n} d \mu-\int_{\mathbb{R}^{n} \times \Omega} \psi d \mu-\sup _{\mathbb{R}^{n} \times \Omega}\left(L_{n}-L-\psi\right)\right) \\
& =\int_{\mathbb{R}^{n} \times \Omega} L_{n} d \mu+\sup _{\psi \in C_{\gamma}^{0}\left(\mathbb{R}^{n} \times \Omega\right)} \int_{\mathbb{R}^{n} \times \Omega}\left(-\psi-\sup _{\mathbb{R}^{n} \times \Omega}\left(L_{n}-L-\psi\right)\right) d \mu .
\end{aligned}
$$

If we take $\psi=0$ in 19$)$ we have

$$
h^{*}(\mu) \geq \int_{\mathbb{R}^{n} \times \Omega} L_{n} d \mu+\int_{\mathbb{R}^{n} \times \Omega}\left(-\sup _{\mathbb{R}^{n} \times \Omega}\left(L_{n}-L\right)\right) d \mu \geq \int_{\mathbb{R}^{n} \times \Omega} L_{n} d \mu .
$$


Thus using the monotone convergence theorem we get

$$
h^{*}(\mu) \geq \int_{\mathbb{R}^{n} \times \Omega} L d \mu .
$$

In order to get the other inequality we can rewrite $(19)$ as follows

$$
\begin{aligned}
h^{*}(\mu)= & \int_{\mathbb{R}^{n} \times \Omega} L_{n} d \mu+\sup _{\psi \in C_{\gamma}^{0}} \int_{\mathbb{R}^{n} \times \Omega}\left((S-\psi-S)-\sup _{\mathbb{R}^{n} \times \Omega}(S-\psi)\right) d \mu \\
= & \int_{\mathbb{R}^{n} \times \Omega} L_{n} d \mu+\sup _{\psi \in C_{\gamma}^{0}} \int_{\mathbb{R}^{n} \times \Omega}\left((S-\psi)-\sup _{\mathbb{R}^{n} \times \Omega}(S-\psi)\right) d \mu \\
& -\int_{\mathbb{R}^{n} \times \Omega} S d \mu,
\end{aligned}
$$

where $S=L_{n}-L$. Since $\mu \in \mathcal{M}_{1}$, we have $\int_{\mathbb{R}^{n} \times \Omega}\left((S-\psi)-\sup _{\mathbb{R}^{n} \times \Omega}(S-\psi)\right) d \mu \leq 0$. Therefore

$$
h^{*}(\mu) \leq \int_{\mathbb{R}^{n} \times \Omega} L_{n} d \mu-\int_{\mathbb{R}^{n} \times \Omega} S d \mu=\int_{\mathbb{R}^{n} \times \Omega} L d \mu .
$$

If $\mu \notin \mathcal{M}_{1}$, we have two possibilities. First, if $\mu \nsupseteq 0$ then we can find a positive function $\psi \in C_{\gamma}^{0}\left(\mathbb{R}^{n} \times \Omega\right)$ such that $\int \psi d \mu<0$. Define $\psi_{n}=n \psi \in C_{\gamma}^{0}\left(\mathbb{R}^{n} \times \Omega\right)$, then

$$
\begin{aligned}
h^{*}(\mu) & \geq\left(-\int_{\mathbb{R}^{n} \times \Omega} \psi_{n} d \mu-\sup _{\mathbb{R}^{n} \times \Omega}\left(-\psi_{n}-L\right)\right) \\
& =n\left(\int_{\mathbb{R}^{n} \times \Omega}-\psi d \mu+\inf _{\mathbb{R}^{n} \times \Omega}\left(\psi+\frac{1}{n} L\right)\right) \rightarrow+\infty,
\end{aligned}
$$

when $n \rightarrow \infty$.

On the other hand, if $\mu \geq 0$ but $\int d \mu \neq 1$ we take $\phi=k \in \mathbb{R}$, then

$$
\begin{aligned}
h^{*}(\mu) & \geq\left(-\int_{\mathbb{R}^{n} \times \Omega} k d \mu-\sup _{\mathbb{R}^{n} \times \Omega}(-k-L)\right) \\
& =k\left(1-\int_{\mathbb{R}^{n} \times \Omega} d \mu\right)+\inf _{\mathbb{R}^{n} \times \Omega} L \rightarrow+\infty
\end{aligned}
$$

when $k \rightarrow \pm \infty$, because $L \geq 0$.

Now we compute the Legendre transform of $g$. As $g$ is concave we compute its Legendre-Fenchel transform using (17). First we suppose $\mu \in \mathcal{M}_{2}$. In this case we have two possibilities, if $\phi \in \mathcal{C}$ then

$$
-\int_{\mathbb{R}^{n} \times \Omega} \phi d \mu-g(\phi)=0,
$$

São Paulo J.Math.Sci. 6, 2 (2012), 301334 
else if, $\phi \notin \mathcal{C}$ then

$$
-\int_{\mathbb{R}^{n} \times \Omega} \phi d \mu-g(\phi)=-\int_{\mathbb{R}^{n} \times \Omega} \phi d \mu-(-\infty)=+\infty
$$

thus $g^{*}(\mu)=0$.

Otherwise, if $\mu \notin \mathcal{M}_{2}$ there exists $\phi(v, \omega)=v \cdot D_{x} \varphi(0, \omega) \in \mathcal{C}$ such that $\int_{\mathbb{R}^{n} \times \Omega} \phi d \mu>0$. Define $\phi_{\lambda}=\lambda v \cdot D_{x} \varphi(0, \omega) \in \mathcal{C}$ then

$$
g^{*}(\mu) \leq\left(-\int_{\mathbb{R}^{n} \times \Omega} \phi_{\lambda} d \mu-g\left(\phi_{\lambda}\right)\right)=-\lambda \int_{\mathbb{R}^{n} \times \Omega} \phi d \mu \rightarrow-\infty
$$

when $\lambda \rightarrow+\infty$.

Remark 3. Observe that

$$
\min _{\mathcal{M}_{1} \cap \mathcal{M}_{2}} \int_{\mathbb{R}^{n} \times \Omega} L(0, v, \omega) d \mu(v, \omega)=\min _{\mathcal{M}}\left(h^{*}(\mu)-g^{*}(\mu)\right) .
$$

In fact,

$$
h^{*}(\mu)-g^{*}(\mu)= \begin{cases}\int L(0, v, \omega) d \mu(v, \omega)-0 & \text { if } \mu \in \mathcal{M}_{1} \cap \mathcal{M}_{2} \\ \int L(0, v, \omega) d \mu(v, \omega)-(-\infty) & \text { if } \mu \in \mathcal{M}_{1} \text { and } \mu \notin \mathcal{M}_{2} \\ +\infty-(0) & \text { if } \mu \notin \mathcal{M}_{1} \text { and } \mu \in \mathcal{M}_{2} \\ +\infty-(-\infty) & \text { if } \mu \notin \mathcal{M}_{1} \text { and } \mu \notin \mathcal{M}_{2}\end{cases}
$$

Lemma 18. The function

$$
h(\phi)=\sup _{\mathbb{R}^{n} \times \Omega}(\phi(v, \omega)-L(0, v, \omega))
$$

is continuous.

Proof. Let $\phi_{0}$ be an arbitrary, but fixed, function in $C_{\gamma}^{0}\left(\mathbb{R}^{n} \times \Omega\right)$. Suppose $\phi_{n} \rightarrow \phi_{0}$, that is $\left\|\phi_{n}-\phi_{0}\right\|_{\gamma} \rightarrow 0$. $\rightarrow$ Let $B_{\varepsilon}\left(\phi_{0}\right)=\left\{\phi \in C_{\gamma}^{0}\left(\mathbb{R}^{n} \times \Omega\right) \mid\left\|\phi_{n}-\phi\right\|_{\gamma}<\varepsilon\right\}$ be the ball of radius $\varepsilon$ centered in $\phi_{0}$. Take $\phi \in B_{\varepsilon}\left(\phi_{0}\right)$. Since, $\lim _{|v| \rightarrow \infty} \frac{|v|}{\gamma(v)}=0, \lim _{|v| \rightarrow \infty} \frac{L(0, v, \omega)}{\gamma(v)}=$ $+\infty$ and $\lim _{|v| \rightarrow \infty} \frac{\left|\phi_{0}(v, \omega)\right|}{\gamma(v)} \rightarrow 0$ uniformly on $\omega \in \Omega$, given $\delta, M>0$, there exists $R>0$ such that

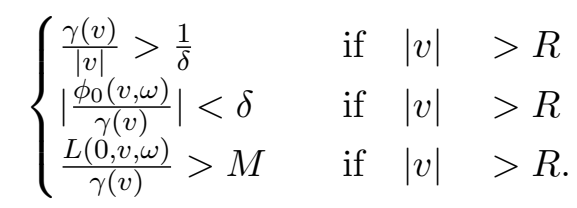


Then, for $|v|>R$,

$$
\begin{aligned}
-\phi(v, \omega)- & L(0, v, \omega)= \\
& \left\{\frac{-\phi(v, \omega)+\phi_{0}(v, \omega)}{\gamma(v)}+\frac{-\phi_{0}(v, \omega)}{\gamma(v)}-\frac{L(0, v, \omega)}{\gamma(v)}\right\} \gamma(v)< \\
& \frac{\gamma(v)}{|v|}(\varepsilon+\delta-M)|v| \rightarrow-\infty
\end{aligned}
$$

when $|v| \rightarrow+\infty$.

As

$$
\left\|\phi_{n}-\phi_{0}\right\|_{\gamma}=\sup _{\mathbb{R}^{n} \times \Omega} \frac{\left|\left(\phi_{n}-\phi_{0}\right)(v, \omega)\right|}{\gamma(v)} \rightarrow 0
$$

we have that, for $n$ big enough, we can choose $R$ in such way that

$$
h(\phi)=\sup _{\{|v| \leq R\} \times \Omega}(-\phi(v, \omega)-L(0, v, \omega)),
$$

and

$$
h\left(\phi_{n}\right)=\sup _{\{|v| \leq R\} \times \Omega}\left(-\phi_{n}(v, \omega)-L(0, v, \omega)\right) .
$$

Since the convergence $-\phi_{n}-L(0, v, \omega) \rightarrow-\phi_{0}-L(0, v, \omega)$ is uniform on the compact $\{|v| \leq R\} \times \Omega$, we have

$$
\begin{gathered}
\lim _{n \rightarrow \infty} h\left(\phi_{n}\right)=\lim _{n \rightarrow \infty} \sup _{\{|v| \leq R\} \times \Omega}\left(-\phi_{n}(v, \omega)-L(0, v, \omega)\right)= \\
=\sup _{\{|v| \leq R\} \times \Omega} \lim _{n \rightarrow \infty}\left(-\phi_{n}(v, \omega)-L(0, v, \omega)\right)=h\left(\phi_{0}\right) .
\end{gathered}
$$

Thus the lemma is proved.

The last ingredient of the duality is the Legendre-Fenchel-Rockafellar Theorem, see for instance [Vil03].

Theorem 19. (Legendre-Fenchel-Rockafellar) Let $E$ be a locally convex Hausdorff topological vectorial space over $\mathbb{R}$ with dual $E^{*}$. Suppose that $h: E \rightarrow(-\infty,+\infty]$ is convex and lower semicontinuous and $g: E^{*} \rightarrow$ $[-\infty,+\infty)$ is concave and upper semicontinuous. Then

$$
\min _{E^{*}}\left(h^{*}-g^{*}\right)=\sup _{E}(g-h),
$$

provided that $h$ or $g$ is continuous at some point where both functions are finite. It is part of the theorem that the left hand side is a minimum.

Lemma 20. Define the functional, $S(\phi)=g(\phi)-h(\phi)$. Then $S$ is uniformly continuous in the interior of $\mathcal{C}$.

São Paulo J.Math.Sci. 6, 2 (2012), 301334 
Proof. In fact, given $\varepsilon>0$, if $\left\|\phi_{1}-\phi_{2}\right\|_{\gamma}<\varepsilon$, that is, $-\varepsilon \gamma(v)<\phi_{1}(v, w)-$ $\phi_{2}(v, w)<\varepsilon \gamma(v)$, for all $(v, w)$, then

$$
\left|S\left(\phi_{1}\right)-S\left(\phi_{2}\right)\right|<\{\inf \gamma(v)\} \varepsilon \text {. }
$$

In particular

$$
\sup _{\phi \in \mathcal{C}} g(\phi)-h(\phi)=\sup _{\substack{\phi=v \cdot D_{x} \varphi(0, \omega) \\ \varphi \in C_{s}^{1}}} g(\phi)-h(\phi) .
$$

\section{B. Proof of Proposition 4}

Proof of Proposition 4. We must prove that the function $u_{\alpha}$ is stationary. Since $L \geq 0, u_{\alpha}$ is well defined as an infimum. On the other hand the stationarity is an easy consequence of the correspondence between the set of all globally Lipschitz trajectories with initial condition $x(0)=x$ and the set of all globally Lipschitz trajectories with initial condition $y(0)=0$, given by, $\{x(t)\} \rightarrow\{y(t)=x(t)-x\}$. In fact,

$$
\begin{aligned}
u_{\alpha}\left(0, \tau_{x} \omega\right) & =\inf _{y(0)=0} \int_{0}^{+\infty} e^{-\alpha t} L\left(y(t), \dot{y}(t), \tau_{x} \omega\right) d t \\
& =\inf _{x(0)=x} \int_{0}^{+\infty} e^{-\alpha t} L((x(t)-x)+x, \dot{x}(t), \omega) d t=u_{\alpha}(x, \omega) .
\end{aligned}
$$

In order to prove that $u_{\alpha}$ is a viscosity solution in $\omega$, let $\varphi: \mathbb{R}^{n} \times \Omega \rightarrow \mathbb{R}$ be a stationary function such that $u_{\alpha}(0, \omega)-\varphi(0, \omega)$ has a local minimum (resp. maximum) in $\omega_{\varphi} \in \Omega$ and $u_{\alpha}\left(0, \omega_{\varphi}\right)-\varphi\left(0, \omega_{\varphi}\right)=0$.

Consider a trajectory satisfying $x(0)=0$ such that $x(t)$ is a finite time minimizing, globally Lipschitz trajectory, for the dynamic programing principle (12), that is,

$$
u_{\alpha}\left(0, \omega_{\varphi}\right)=\int_{0}^{T} e^{-\alpha t} L\left(x(t), \dot{x}(t), \omega_{\varphi}\right) d t+e^{-\alpha T} u_{\alpha}\left(x(T), \omega_{\varphi}\right),
$$

for $T$ small enough.

Suppose that $\mathcal{H}\left(\varphi, \omega_{\varphi}\right)<0$, by continuity there is a neighborhood $B$ of $\omega_{\varphi}$ in $\Omega$ and $\delta>0$ such that $\mathcal{H}(\varphi, \omega)<-\delta$ for all $\omega \in B$. Since $\mathcal{H}(\varphi, \omega)=$ $H\left(0, D_{x} \varphi(0, \omega), \omega\right)+\alpha \varphi(0, \omega)$ we have $-v D_{x} \varphi(0, \omega)-L(0, v, \omega)+\alpha \varphi(0, \omega)<$ $-\delta$, for all $\omega \in B$ and $v \in \mathbb{R}^{n}$. If we choose $v=\dot{x}(t)$ and $\omega=\tau_{x(t)} \omega_{\varphi}$ then

$$
\dot{x}(t) D_{x} \varphi\left(0, \tau_{x(t)} \omega_{\varphi}\right)+L\left(x(t), \dot{x}(t), \omega_{\varphi}\right)-\alpha \varphi\left(x(t), \omega_{\varphi}\right)>\delta,
$$

for $0<t<T$. 
Integrating this expression and using $\frac{d}{d t} \varphi(x(t), \omega)=\dot{x}(t) D_{x} \varphi\left(0, \tau_{x(t)} \omega\right)$ we get,

$\varphi\left(0, \tau_{x(T)} \omega_{\varphi}\right)-\varphi\left(0, \omega_{\varphi}\right)+\int_{0}^{T} L\left(x(t), \dot{x}(t), \omega_{\varphi}\right) d t-\alpha \int_{0}^{T} \varphi\left(x(t), \omega_{\varphi}\right) d t>\delta T$.

Since $u_{\alpha}(0, \omega) \geq \varphi(0, \omega)$ in $B$ and $u_{\alpha}\left(0, \omega_{\varphi}\right)=\varphi\left(0, \omega_{\varphi}\right)$, we have

$u_{\alpha}\left(0, \tau_{x(T)} \omega_{\varphi}\right)-u_{\alpha}\left(0, \omega_{\varphi}\right)+\int_{0}^{T} L\left(x(t), \dot{x}(t), \omega_{\varphi}\right) d t-\alpha \int_{0}^{T} \varphi\left(x(t), \omega_{\varphi}\right) d t>\delta T$.

Using 20 in the last inequality we get,

$$
\begin{aligned}
\left(1-e^{-\alpha T}\right) u_{\alpha}\left(0, \tau_{x(T)} \omega_{\varphi}\right)+\int_{0}^{T}\left(1-e^{-\alpha t}\right) & L\left(x(t), \dot{x}(t), \omega_{\varphi}\right) d t \\
& -\alpha \int_{0}^{T} \varphi\left(x(t), \omega_{\varphi}\right) d t>\delta T .
\end{aligned}
$$

Writing

$$
\begin{gathered}
u_{\alpha}\left(0, \tau_{x(T)} \omega_{\varphi}\right)+\frac{T}{\left(1-e^{-\alpha T}\right)} \frac{1}{T} \int_{0}^{T}\left(1-e^{-\alpha t}\right) L\left(x(t), \dot{x}(t), \omega_{\varphi}\right) d t- \\
\alpha \frac{T}{\left(1-e^{-\alpha T}\right)} \frac{1}{T} \int_{0}^{T} \varphi\left(x(t), \omega_{\varphi}\right) d t>\delta \frac{T}{1-e^{-\alpha T}}
\end{gathered}
$$

and using $\lim _{T \rightarrow 0} \frac{T}{1-e^{-\alpha T}}=\frac{1}{\alpha}$, we get

$$
u_{\alpha}\left(0, \omega_{\varphi}\right)-\varphi\left(0, \omega_{\varphi}\right)>\frac{\delta}{\alpha}
$$

contradicting $u_{\alpha}\left(0, \omega_{\varphi}\right)=\varphi\left(0, \omega_{\varphi}\right)$.

The proof for the maximum case is analogous and so the theorem is proved.

\section{Proof of Theorem 14}

In this last appendix we give a proof of Theorem 14. Before that we need to establish some additional results. We note here that we will be using the techniques in [EG01] (see also [BG08]) adapted to the stationary setting.

Remark 4. Let $u_{\alpha}$ be a viscosity solution in $\omega$ of $(10)$, then, because it is also a viscosity solution in $x$ (Proposition 2) and it is Lipschitz, $D_{x} u_{\alpha}\left(0, \tau_{y} \omega\right)$ is defined Lebesgue almost everywhere and

$$
H\left(y, D_{x} u_{\alpha}(y, \omega), \omega\right)+\alpha u_{\alpha}(y, \omega)=0,
$$

for Lebesgue almost all $y \in \mathbb{R}^{n}$.

São Paulo J.Math.Sci. 6, 2 (2012), 301334 
For any probability measure $\mu$, we can define a new measure of probability $\tilde{\mu}$ in $\mathbb{R}^{n} \times \Omega$ given by,

$$
\int_{\mathbb{R}^{n} \times \Omega} \psi(p, \omega) d \tilde{\mu}(p, \omega)=\int_{\mathbb{R}^{n} \times \Omega} \psi\left(-D_{v} L(0, v, \omega), \omega\right) d \mu(v, \omega) .
$$

In this case, the integral holonomy constraint can be rewritten as

$$
\int_{\mathbb{R}^{n} \times \Omega} D_{p} H(0, p, \omega) \cdot D_{x} \varphi(0, \omega) d \tilde{\mu}(p, \omega)=0,
$$

$\forall \varphi \in C_{s}^{1}\left(\mathbb{R}^{n} \times \Omega\right)$.

Theorem 21. Let $\mu_{\alpha}$ be a holonomic discounted stationary Mather measure. Denote the projection in the coordinate $\omega$ of $\mu_{\alpha}$ by $\theta_{\alpha}$, that is

$$
\int_{\Omega} \varphi(\omega) d \theta_{\alpha}=\int_{\mathbb{R}^{n} \times \Omega} \varphi(\omega) d \mu_{\alpha}
$$

If $u_{\alpha}$ is a viscosity solution of $(10)$, then $D_{x} u_{\alpha}(0, \omega)$ exists $\theta_{\alpha}$-a.e, and $\tilde{\mu}_{\alpha}$-a.e, $p=-D_{x} u_{\alpha}(0, \omega)$.

Proof. By the strict uniform continuity of $H$ there exists $\gamma>0$ such that for any $p, q, y \in \mathbb{R}^{n}$ and $\omega \in \Omega$ we have

$$
H\left(0, p, \tau_{y} \omega\right) \geq H\left(0, q, \tau_{y} \omega\right)+D_{p} H\left(0, q, \tau_{y} \omega\right)(p-q)+\frac{\gamma}{2}|p-q|^{2} .
$$

Let $u^{\varepsilon}=u * \eta$, by Remark 4 , for almost every $\omega$ and $y$, let $p=D_{x} u_{\alpha}\left(0, \tau_{y} \omega\right)$ and $q=D_{x} u_{\alpha}^{\varepsilon}(0, \omega)$. Then

$$
\begin{aligned}
H\left(0, D_{x} u_{\alpha}\left(0, \tau_{y} \omega\right),\right. & \left.\tau_{y} \omega\right) \geq H\left(0, D_{x} u_{\alpha}^{\varepsilon}(0, \omega), \tau_{y} \omega\right) \\
& +D_{p} H\left(0, D_{x} u_{\alpha}^{\varepsilon}(0, \omega), \tau_{y} \omega\right)\left(D_{x} u_{\alpha}\left(0, \tau_{y} \omega\right)-D_{x} u_{\alpha}^{\varepsilon}(0, \omega)\right) \\
& +\frac{\gamma}{2}\left|D_{x} u_{\alpha}\left(0, \tau_{y} \omega\right)-D_{x} u_{\alpha}^{\varepsilon}(0, \omega)\right|^{2} .
\end{aligned}
$$

Multiplying by $\eta^{\varepsilon}(y)$ and integrating we get

$$
\begin{aligned}
& \int_{\mathbb{R}^{n}} H\left(0, D_{x} u_{\alpha}^{\varepsilon}(0, \omega), \tau_{y} \omega\right) \eta^{\varepsilon}(y) d y+\int_{\mathbb{R}^{n}} \frac{\gamma}{2} \mid D_{x} u_{\alpha}\left(0, \tau_{y} \omega\right) \\
& -\left.D_{x} u_{\alpha}^{\varepsilon}(0, \omega)\right|^{2} \eta^{\varepsilon}(y) d y \\
& \leq \int_{\mathbb{R}^{n}} H\left(0, D_{x} u_{\alpha}\left(0, \tau_{y} \omega\right), \tau_{y} \omega\right) \eta^{\varepsilon}(y) d y \\
& +\int_{\mathbb{R}^{n}} D_{p} H\left(0, D_{x} u_{\alpha}^{\varepsilon}(0, \omega), \tau_{y} \omega\right)\left[D_{x} u_{\alpha}^{\varepsilon}(0, \omega)-D_{x} u_{\alpha}\left(0, \tau_{y} \omega\right)\right] \eta^{\varepsilon}(y) d y .
\end{aligned}
$$


Remark 4 implies that, $H\left(y, D_{x} u_{\alpha}(y, \omega), \omega\right)=-\alpha u_{\alpha}(y, \omega)$ almost everywhere $y$. Thus

$$
\int_{\mathbb{R}^{n}} H\left(0, D_{x} u_{\alpha}^{\varepsilon}\left(0, \tau_{y} \omega\right), \tau_{y} \omega\right) \eta^{\varepsilon}(y) d y+\beta^{\varepsilon}(\omega) \leq-\alpha u_{\alpha}^{\varepsilon}(0, \omega)+o_{\omega}(\varepsilon)
$$

where

$$
\beta^{\varepsilon}(\omega)=\int_{\mathbb{R}^{n}} \frac{\gamma}{4}\left|D_{x} u_{\alpha}\left(0, \tau_{y} \omega\right)-D_{x} u_{\alpha}^{\varepsilon}\left(0, \tau_{y} \omega\right)\right|^{2} \eta^{\varepsilon}(y) d y .
$$

On the other hand, the convexity of $H$, implies that,

$$
\begin{aligned}
& \int_{\mathbb{R}^{n} \times \Omega} \frac{\gamma}{2}\left|D_{x} u_{\alpha}^{\varepsilon}(0, \omega)-p\right|^{2} d \tilde{\mu}_{\alpha}(p, \omega) \\
& \leq \int_{\mathbb{R}^{n} \times \Omega}\left[H\left(0, D_{x} u_{\alpha}^{\varepsilon}(0, \omega), \omega\right)-H(0, p, \omega)\right. \\
& \left.\quad-D_{p} H(0, p, \omega)\left(D_{x} u_{\alpha}^{\varepsilon}(0, \omega)-p\right)\right] d \tilde{\mu}_{\alpha}(p, \omega) \\
& =\int_{\mathbb{R}^{n} \times \Omega} H\left(0, D_{x} u_{\alpha}^{\varepsilon}(0, \omega), \omega\right) d \tilde{\mu}_{\alpha}(p, \omega) \\
& -\int_{\mathbb{R}^{n} \times \Omega}\left[H(0, p, \omega)+D_{p} H(0, p, \omega) D_{x} u_{\alpha}^{\varepsilon}(0, \omega)-D_{p} H(0, p, \omega) p\right] d \tilde{\mu}_{\alpha}(p, \omega) \\
& =\int_{\mathbb{R}^{n} \times \Omega} H\left(0, D_{x} u_{\alpha}^{\varepsilon}(0, \omega), \omega\right)+L\left(0,-D_{p} H(0, p, \omega), \omega\right) d \tilde{\mu}_{\alpha}(p, \omega) \\
& =\int_{\mathbb{R}^{n} \times \Omega} H\left(0, D_{x} u_{\alpha}^{\varepsilon}(0, \omega), \omega\right) d \tilde{\mu}_{\alpha}(p, \omega)+\bar{H}_{\alpha} .
\end{aligned}
$$

Integrating (21) with respect to $\tilde{\mu}$ and adding $(22)$, we get

$$
\int_{\mathbb{R}^{n} \times \Omega} \frac{\gamma}{2}\left|D_{x} u_{\alpha}^{\varepsilon}(0, \omega)-p\right|^{2} d \tilde{\mu}_{\alpha}(p, \omega)+\int_{\Omega} \beta^{\varepsilon}(\omega) d \theta(\omega)<o(\varepsilon) .
$$

So, $\theta_{\alpha}$ almost everywhere we have $D_{x} u_{\alpha}(0, \omega)=\lim _{\varepsilon \rightarrow 0} D_{x} u_{\alpha}^{\varepsilon}(0, \omega)$, in particular $p=D_{x} u_{\alpha}(0, \omega)$ in the support of $\tilde{\mu}$.

Theorem 22. Let $\mu_{\alpha}$ be a holonomic Mather measure for the discounted stationary Mather problem. If $u_{\alpha}$ is a viscosity solution of (10), then for each $h \in \mathbb{R}$,

$$
\left|u_{\alpha}(h, \omega)-2 u_{\alpha}(0, \omega)+u_{\alpha}(-h, \omega)\right| \leq C|h|^{2},
$$

$\theta$ almost everywhere.

Proof. If $h \neq 0$ then we define,

$$
\tilde{u}_{\alpha}(x, \omega)=u_{\alpha}(x+h, \omega) \text { and } \hat{u}_{\alpha}(x, \omega)=u_{\alpha}(x-h, \omega),
$$


and $\tilde{u}_{\alpha}^{\varepsilon}(x, \omega)$ and $\hat{u}_{\alpha}^{\varepsilon}(x, \omega)$, the corresponding smoothings (see Remark 1).

Remember that

$$
H\left(h, D_{x} \tilde{u}_{\alpha}^{\varepsilon}(0, \omega), \omega\right)+\alpha \tilde{u}_{\alpha}^{\varepsilon}(0, \omega) \leq c \varepsilon,
$$

and

Thus,

$$
H\left(-h, D_{x} \hat{u}_{\alpha}^{\varepsilon}(0, \omega), \omega\right)+\alpha \hat{u}_{\alpha}^{\varepsilon}(0, \omega) \leq c \varepsilon
$$

$$
\begin{aligned}
H(0, & \left.D_{x} \tilde{u}_{\alpha}^{\varepsilon}(0, \omega), \omega\right)-2 H\left(0, D_{x} u_{\alpha}(0, \omega), \omega\right)+H\left(0, D_{x} \hat{u}_{\alpha}^{\varepsilon}(0, \omega), \omega\right) \\
= & H\left(0, D_{x} \tilde{u}_{\alpha}^{\varepsilon}(0, \omega), \omega\right)-H\left(h, D_{x} \tilde{u}_{\alpha}^{\varepsilon}(0, \omega), \omega\right)+H\left(h, D_{x} \tilde{u}_{\alpha}^{\varepsilon}(0, \omega), \omega\right) \\
& +\alpha \tilde{u}_{\alpha}^{\varepsilon}(0, \omega)-\alpha \tilde{u}_{\alpha}^{\varepsilon}(0, \omega)+2 \alpha u_{\alpha}(0, \omega)-\alpha \hat{u}_{\alpha}^{\varepsilon}(0, \omega) \\
& +\alpha \hat{u}_{\alpha}^{\varepsilon}(0, \omega)+H\left(-h, D_{x} \hat{u}_{\alpha}^{\varepsilon}(0, \omega), \omega\right)-H\left(-h, D_{x} \hat{u}_{\alpha}^{\varepsilon}(0, \omega), \omega\right) \\
& +H\left(0, D_{x} \hat{u}_{\alpha}^{\varepsilon}(0, \omega), \omega\right) \leq 2 c \varepsilon-\alpha\left(\tilde{u}_{\alpha}^{\varepsilon}(0, \omega)-2 u_{\alpha}(0, \omega)+\hat{u}_{\alpha}^{\varepsilon}(0, \omega)\right) \\
& -\left(D_{x} H\left(0, D_{x} \tilde{u}_{\alpha}^{\varepsilon}(0, \omega), \omega\right)-D_{x} H\left(0, D_{x} \hat{u}_{\alpha}^{\varepsilon}(0, \omega), \omega\right)\right) h+O\left(|h|^{2}\right) .
\end{aligned}
$$

On the other hand the convexity of $H$ implies that

$$
\begin{aligned}
H\left(0, D_{x} \tilde{u}_{\alpha}^{\varepsilon}(0, \omega), \omega\right) \geq & H\left(0, D_{x} u_{\alpha}(0, \omega), \omega\right) \\
& +D_{p} H\left(0, D_{x} u_{\alpha}(0, \omega), \omega\right)\left(D_{x} \tilde{u}_{\alpha}^{\varepsilon}(0, \omega)-D_{x} u_{\alpha}(0, \omega)\right) \\
& +\frac{\gamma}{2}\left|D_{x} \tilde{u}_{\alpha}^{\varepsilon}(0, \omega)-D_{x} u_{\alpha}(0, \omega)\right|^{2}
\end{aligned}
$$

and

$$
\begin{aligned}
H\left(0, D_{x} \hat{u}_{\alpha}^{\varepsilon}(0, \omega), \omega\right) \geq & H\left(0, D_{x} u_{\alpha}(0, \omega), \omega\right) \\
& +D_{p} H\left(0, D_{x} u_{\alpha}(0, \omega), \omega\right)\left(D_{x} \hat{u}_{\alpha}^{\varepsilon}(0, \omega)-D_{x} u_{\alpha}(0, \omega)\right) \\
& +\frac{\gamma}{2}\left|D_{x} \hat{u}_{\alpha}^{\varepsilon}(0, \omega)-D_{x} u_{\alpha}(0, \omega)\right|^{2} .
\end{aligned}
$$

Adding these two formulas we obtain the following inequality:

$$
\begin{aligned}
& \frac{\gamma}{2}\left(\left|D_{x} \tilde{u}_{\alpha}^{\varepsilon}(0, \omega)-D_{x} u_{\alpha}(0, \omega)\right|^{2}+\left|D_{x} \hat{u}_{\alpha}^{\varepsilon}(0, \omega)-D_{x} u_{\alpha}(0, \omega)\right|^{2}\right) \\
& +D_{p} H\left(0, D_{x} u_{\alpha}(0, \omega), \omega\right)\left(D_{x} \tilde{u}_{\alpha}^{\varepsilon}(0, \omega)-2 D_{x} u_{\alpha}(0, \omega)+D_{x} \hat{u}_{\alpha}^{\varepsilon}(0, \omega)\right) \\
& \leq\left(H\left(0, D_{x} \tilde{u}_{\alpha}^{\varepsilon}(0, \omega), \omega\right)-2 H\left(0, D_{x} u_{\alpha}(0, \omega), \omega\right)+H\left(0, D_{x} \hat{u}_{\alpha}^{\varepsilon}(0, \omega), \omega\right)\right) .
\end{aligned}
$$

By 23) we have,

$$
\begin{aligned}
& \frac{\gamma}{2}\left(\left|D_{x} \tilde{u}_{\alpha}^{\varepsilon}(0, \omega)-D_{x} u_{\alpha}(0, \omega)\right|^{2}+\left|D_{x} \hat{u}_{\alpha}^{\varepsilon}(0, \omega)-D_{x} u_{\alpha}(0, \omega)\right|^{2}\right) \\
& +D_{p} H\left(0, D_{x} u_{\alpha}(0, \omega), \omega\right)\left(D_{x} \tilde{u}_{\alpha}^{\varepsilon}(0, \omega)-2 D_{x} u_{\alpha}(0, \omega)+D_{x} \hat{u}_{\alpha}^{\varepsilon}(0, \omega)\right) \\
& \leq 2 c \varepsilon-\alpha\left(\tilde{u}_{\alpha}^{\varepsilon}(0, \omega)-2 u_{\alpha}(0, \omega)+\hat{u}_{\alpha}^{\varepsilon}(0, \omega)\right) \\
& -\left(D_{x} H\left(0, D_{x} \tilde{u}_{\alpha}^{\varepsilon}(0, \omega), \omega\right)-D_{x} H\left(0, D_{x} \hat{u}_{\alpha}^{\varepsilon}(0, \omega), \omega\right)\right) h+O\left(|h|^{2}\right) .
\end{aligned}
$$


Or equivalently,

$$
\begin{aligned}
& \frac{\gamma}{2}\left(\left|D_{x} \tilde{u}_{\alpha}^{\varepsilon}(0, \omega)-D_{x} u_{\alpha}(0, \omega)\right|^{2}+\left|D_{x} \hat{u}_{\alpha}^{\varepsilon}(0, \omega)-D_{x} u_{\alpha}(0, \omega)\right|^{2}\right) \\
& +D_{p} H\left(0, D_{x} u_{\alpha}(0, \omega), \omega\right)\left(D_{x} \tilde{u}_{\alpha}^{\varepsilon}(0, \omega)-2 D_{x} u_{\alpha}(0, \omega)+D_{x} \hat{u}_{\alpha}^{\varepsilon}(0, \omega)\right) \\
& +\alpha\left(\tilde{u}_{\alpha}^{\varepsilon}(0, \omega)-2 u_{\alpha}(0, \omega)+\hat{u}_{\alpha}^{\varepsilon}(0, \omega)\right) \\
& \leq 2 c \varepsilon-\left(D_{x} H\left(0, D_{x} \tilde{u}_{\alpha}^{\varepsilon}(0, \omega), \omega\right)-D_{x} H\left(0, D_{x} \hat{u}_{\alpha}^{\varepsilon}(0, \omega), \omega\right)\right)(h)+O\left(|h|^{2}\right) .
\end{aligned}
$$

Define, $\beta^{\varepsilon}(x, \omega)=\tilde{u}_{\alpha}^{\varepsilon}(x, \omega)-2 u_{\alpha}(x, \omega)+\hat{u}_{\alpha}^{\varepsilon}(x, \omega)$, so (24) can be rewritten as

$$
\begin{aligned}
& \frac{\gamma}{2}\left(\left|D_{x} \tilde{u}_{\alpha}^{\varepsilon}(0, \omega)-D_{x} u_{\alpha}(0, \omega)\right|^{2}+\left|D_{x} \hat{u}_{\alpha}^{\varepsilon}(0, \omega)-D_{x} u_{\alpha}(0, \omega)\right|^{2}\right) \\
& +D_{p} H\left(0, D_{x} u_{\alpha}(0, \omega), \omega\right) D_{x} \beta^{\varepsilon}(0, \omega)+\alpha \beta^{\varepsilon}(0, \omega) \\
& \leq 2 c \varepsilon-\left(D_{x} H\left(0, D_{x} \tilde{u}_{\alpha}^{\varepsilon}(0, \omega), \omega\right)-D_{x} H\left(0, D_{x} \hat{u}_{\alpha}^{\varepsilon}(0, \omega), \omega\right)\right)(h)+O\left(|h|^{2}\right)
\end{aligned}
$$

Applying the inequality

$$
\begin{aligned}
& \left\|\left(D_{x} H\left(0, D_{x} \tilde{u}_{\alpha}^{\varepsilon}(0, \omega), \omega\right)-D_{x} H\left(0, D_{x} \hat{u}_{\alpha}^{\varepsilon}(0, \omega), \omega\right)\right) h\right\| \\
\leq & \left\|D^{2} p x H\left(0, D_{x} \tilde{u}_{\alpha}^{\varepsilon}(0, \omega), \omega\right)\right\| \cdot\left|D_{x} \tilde{u}_{\alpha}^{\varepsilon}(0, \omega)-D_{x} \hat{u}_{\alpha}^{\varepsilon}(0, \omega)\right| \cdot|h| \\
\leq & \frac{\gamma}{4}\left|D_{x} \tilde{u}_{\alpha}^{\varepsilon}(0, \omega)-D_{x} \hat{u}_{\alpha}^{\varepsilon}(0, \omega)\right|^{2}+\frac{1}{\gamma}\left|D_{x} \tilde{u}_{\alpha}^{\varepsilon}(0, \omega)-D_{x} \hat{u}_{\alpha}^{\varepsilon}(0, \omega)\right|^{2} \cdot|h|^{2} \\
\leq & \frac{\gamma}{4}\left(\left|D_{x} \tilde{u}_{\alpha}^{\varepsilon}(0, \omega)-D_{x} u_{\alpha}(0, \omega)\right|^{2}+\left|D_{x} \hat{u}_{\alpha}^{\varepsilon}(0, \omega)-D_{x} u_{\alpha}(0, \omega)\right|^{2}\right) \\
& +\frac{1}{\gamma}\left|D_{x} \tilde{u}_{\alpha}^{\varepsilon}(0, \omega)-D_{x} \hat{u}_{\alpha}^{\varepsilon}(0, \omega)\right|^{2} \cdot|h|^{2},
\end{aligned}
$$

to 25) we get,

$$
\begin{aligned}
& \frac{\gamma}{4}\left(\left|D_{x} \tilde{u}_{\alpha}^{\varepsilon}(0, \omega)-D_{x} u_{\alpha}(0, \omega)\right|^{2}+\left|D_{x} \hat{u}_{\alpha}^{\varepsilon}(0, \omega)-D_{x} u_{\alpha}(0, \omega)\right|^{2}\right) \\
& +D_{p} H\left(0, D_{x} u_{\alpha}(0, \omega), \omega\right) D_{x} \beta^{\varepsilon}(0, \omega)+\alpha \beta^{\varepsilon}(0, \omega) \leq 2 C\left(\varepsilon+|h|^{2}\right) .
\end{aligned}
$$


Consider a function $\Psi: \mathbb{R} \rightarrow \mathbb{R}$, such that $\Phi(s)=\Psi^{\prime}(s) \geq 0$. We can multiply 26 by $\Phi\left(\frac{\beta^{\varepsilon}(0, \omega)}{|h|^{2}}\right)$ and integrate with respect to $\theta$,

$$
\begin{aligned}
& \int_{\Omega} \frac{\gamma}{4}\left(\left|D_{x} \tilde{u}_{\alpha}^{\varepsilon}(0, \omega)-D_{x} u_{\alpha}(0, \omega)\right|^{2}\right. \\
& \left.\quad+\left|D_{x} \hat{u}_{\alpha}^{\varepsilon}(0, \omega)-D_{x} u_{\alpha}(0, \omega)\right|^{2}\right) \Phi\left(\frac{\beta^{\varepsilon}(0, \omega)}{|h|^{2}}\right) d \theta \\
& +\int_{\Omega} D_{p} H\left(0, D_{x} u_{\alpha}(0, \omega), \omega\right) D_{x} \beta^{\varepsilon}(0, \omega) \Phi\left(\frac{\beta^{\varepsilon}(0, \omega)}{|h|^{2}}\right) d \theta \\
& +\int_{\Omega} \alpha \beta^{\varepsilon}(0, \omega) \Phi\left(\frac{\beta^{\varepsilon}(0, \omega)}{|h|^{2}}\right) d \theta \leq 2 C\left(\varepsilon+|h|^{2}\right) \int_{\Omega} \Phi\left(\frac{\beta^{\varepsilon}(0, \omega)}{|h|^{2}}\right) d \theta .
\end{aligned}
$$

We have

$$
\begin{aligned}
& |h|^{2} \int_{\Omega} D_{p} H\left(0, D_{x} u_{\alpha}(0, \omega), \omega\right) \frac{1}{|h|^{2}} D_{x} \beta^{\varepsilon}(0, \omega) \Phi\left(\frac{\beta^{\varepsilon}(0, \omega)}{|h|^{2}}\right) d \theta \\
& =\int_{\Omega} D_{p} H\left(0, D_{x} u_{\alpha}(0, \omega), \omega\right) D_{x} \Psi\left(\frac{\beta^{\varepsilon}}{|h|^{2}}\right)(0, \omega) d \theta=0 .
\end{aligned}
$$

Thus, (27) can be restated as,

$$
\begin{aligned}
& \int_{\Omega}\left|D_{x} \tilde{u}_{\alpha}^{\varepsilon}(0, \omega)-D_{x} \hat{u}_{\alpha}^{\varepsilon}(0, \omega)\right|^{2} \Phi\left(\frac{\beta^{\varepsilon}(0, \omega)}{|h|^{2}}\right) d \theta \\
& +\int_{\Omega} \alpha \beta^{\varepsilon}(0, \omega) \Phi\left(\frac{\beta^{\varepsilon}(0, \omega)}{|h|^{2}}\right) d \theta \leq 2 C\left(\varepsilon+|h|^{2}\right) \int_{\Omega} \Phi\left(\frac{\beta^{\varepsilon}(0, \omega)}{|h|^{2}}\right) d \theta .
\end{aligned}
$$

Define, $A_{\lambda}=\left\{\omega \mid \frac{\beta^{\varepsilon}(0, \omega)}{|h|^{2}} \leq-\lambda\right\}$, and consider the function $\Psi$ defined by

$$
\Psi(s)= \begin{cases}s & \text { if } s \leq-\lambda \\ 1 & \text { otherwise }\end{cases}
$$

Fix a positive constant $\gamma$ such that the functions $\bar{u}_{\alpha}(x, \omega)=\tilde{u}_{\alpha}(x, \omega)-$ $\frac{\gamma}{2}|x|^{2}$ and $\bar{u}_{\alpha}^{\varepsilon}(x, \omega)=\tilde{u}_{\alpha}^{\varepsilon}(x, \omega)-\frac{\gamma}{2}|x|^{2}$ are concave. Observe that a point $\omega$ is in $A_{\lambda}$ only if

$$
\bar{u}_{\alpha}^{\varepsilon}(h, \omega)-2 \bar{u}_{\alpha}(0, \omega)+\bar{u}_{\alpha}^{\varepsilon}(-h, \omega) \leq-(\lambda+\gamma)|h|^{2} .
$$

Define $F^{\varepsilon}(t)=\bar{u}_{\alpha}^{\varepsilon}\left(t \frac{h}{|h|}, \omega\right)$. Since $F^{\varepsilon}$ is concave and $\left(F^{\varepsilon}\right)^{\prime \prime} \leq 0$ we have

$$
\bar{u}_{\alpha}^{\varepsilon}(h, \omega)-2 \bar{u}_{\alpha}^{\varepsilon}(0, \omega)+\bar{u}_{\alpha}^{\varepsilon}(-h, \omega) \geq\left(D_{x} \bar{u}_{\alpha}^{\varepsilon}(h, \omega)-D_{x} \bar{u}_{\alpha}^{\varepsilon}(-h, \omega)\right) h .
$$

Subtracting this inequalities we get,

$$
(\lambda+\gamma)|h|^{2} \leq 2\left|\bar{u}_{\alpha}^{\varepsilon}(0, \omega)-\bar{u}_{\alpha}(0, \omega)\right|+\left|D_{x} \bar{u}_{\alpha}^{\varepsilon}(h, \omega)-D_{x} \bar{u}_{\alpha}^{\varepsilon}(-h, \omega)\right||h| .
$$


Since $u_{\alpha}$ is stationary and uniformly Lipschitz continuous we have $\left|\bar{u}_{\alpha}^{\varepsilon}(0, \omega)-\bar{u}_{\alpha}(0, \omega)\right| \leq C \varepsilon$. thus we can choose $\varepsilon$ in such way that

$$
\left|D_{x} \bar{u}_{\alpha}^{\varepsilon}(h, \omega)-D_{x} \bar{u}_{\alpha}^{\varepsilon}(-h, \omega)\right| \geq\left(\frac{\lambda}{2}+\gamma\right)|h|
$$

and

$$
\left|D_{x} u_{\alpha}^{\varepsilon}(h, \omega)-D_{x} u_{\alpha}^{\varepsilon}(-h, \omega)\right| \geq\left(\frac{\lambda}{2}+\gamma\right)|h| .
$$

Using this estimates in (6) we get

$$
\left(\frac{\lambda}{2}+\gamma\right)^{2}|h|^{2} \theta\left(A_{\lambda}\right)-\alpha \lambda|h|^{2} \theta\left(A_{\lambda}\right) \leq 2 C\left(\varepsilon+|h|^{2}\right) \theta\left(A_{\lambda}\right) .
$$

Observe that, if $\theta\left(A_{\lambda}\right)>0$ then the left hand side of this inequality converges to $+\infty$ when $\lambda \rightarrow+\infty$, so there exists a value $\lambda_{0}$ such that $\theta\left(A_{\lambda}\right)=0$, that is, $-\lambda_{0}|h|^{2} \leq \tilde{u}_{\alpha}^{\varepsilon}(x, \omega)-2 u_{\alpha}(x, \omega)+\hat{u}_{\alpha}^{\varepsilon}(x, \omega), \theta$ almost everywhere. The upper bound comes from the semiconcavity of $u_{\alpha}$. Thus there exists $C>0$ such that $\left|u_{\alpha}(h, \omega)-2 u_{\alpha}(0, \omega)+u_{\alpha}(-h, \omega)\right| \leq C|h|^{2}, \theta$ almost everywhere, which completes the proof of the theorem.

Proof of Theorem 14. Let $\theta$ be the projection of $\mu_{\alpha}$. By Theorem 21 . $D_{x} u_{\alpha}(0, \omega)$ exists $\theta$-a.e. On the other hand, fixed $\omega \in \operatorname{supp} \theta, D_{x} u_{\alpha}(y, \omega)$ exists Lebesgue almost everywhere.

We claim that

$$
\left|u_{\alpha}(y, \omega)-u_{\alpha}(0, \omega)+u_{\alpha}(-y, \omega)\right| \leq C|y|^{2} .
$$

This claim is a consequence of Theorem 22 , by choosing $h=y$ and of the semi-concavity of $u_{\alpha}$. In fact, we have

$$
\begin{gathered}
-C|h|^{2} \leq u_{\alpha}(y, \omega)-2 u_{\alpha}(0, \omega)+u_{\alpha}(-y, \omega) \leq C|h|^{2}, \\
u_{\alpha}(y, \omega)-u_{\alpha}(0, \omega)-D_{x} u_{\alpha}(0, \omega) y \leq C|y|^{2},
\end{gathered}
$$

and

$$
u_{\alpha}(-y, \omega)-u_{\alpha}(0, \omega)+D_{x} u_{\alpha}(0, \omega) y \leq C|y|^{2} .
$$

The claim is obtained from $(30)$ and from the difference between $(29)$ and (31).

Let $z \in \mathbb{R}$ be a point such that $|z| \leq 2|y|$. The semi-concavity of $u_{\alpha}$ implies that,

$$
u_{\alpha}(z, \omega) \leq u_{\alpha}(y, \omega)+D_{x} u_{\alpha}(y, \omega)(z-y)+C|z-y|^{2} .
$$

Using, $u_{\alpha}(z, \omega)=u_{\alpha}(0, \omega)+D_{x} u_{\alpha}(0, \omega) z+o\left(|z|^{2}\right)$ and $u_{\alpha}(y, \omega)=u_{\alpha}(0, \omega)+$ $D_{x} u_{\alpha}(0, \omega) y+o\left(|y|^{2}\right)$ in (4) we get

$$
\left(D_{x} u_{\alpha}(0, \omega)-D_{x} u_{\alpha}(y, \omega)\right)(z-y) \leq C|y|^{2} .
$$

São Paulo J.Math.Sci. 6, 2 (2012), 301334 
If we take $z=y+|y| \frac{D_{x} u_{\alpha}(0, \omega)-D_{x} u_{\alpha}(y, \omega)}{\left|D_{x} u_{\alpha}(0, \omega)-D_{x} u_{\alpha}(y, \omega)\right|}$ then we obtain $\mid D_{x} u_{\alpha}(y, \omega)-$ $D_{x} u_{\alpha}(0, \omega)|\leq C| y \mid$.

\section{References}

[BCD97] M. Bardi and I. Capuzzo-Dolcetta. Optimal control and viscosity solutions of Hamilton-Jacobi-Bellman equations. Birkhäuser Boston Inc., Boston, MA, 1997. With appendices by Maurizio Falcone and Pierpaolo Soravia.

[BDLLS08] G. Barles, F. Da Lio, P.-L. Lions, and P. E. Souganidis. Ergodic problems and periodic homogenization for fully nonlinear equations in half-space type domains with Neumann boundary conditions. Indiana Univ. Math. J., 57(5):2355-2375, 2008.

[BG08] A. Biryuk and D. Gomes. An introduction to the Aubry-Mather theory. preprint, 2008.

[CSW05] Luis A. Caffarelli, Panagiotis E. Souganidis, and L. Wang. Homogenization of fully nonlinear, uniformly elliptic and parabolic partial differential equations in stationary ergodic media. Comm. Pure Appl. Math., 58(3):319-361, 2005.

[DS09] Andrea Davini and Antonio Siconolfi. Exact and approximate correctors for stochastic Hamiltonians: the 1-dimensional case. Math. Ann., 345(4):749-782, 2009.

[EG01] L. C. Evans and D. Gomes. Effective Hamiltonians and averaging for Hamiltonian dynamics. I. Arch. Ration. Mech. Anal., 157(1):1-33, 2001.

[FCG08] Italo Capuzzo Dolcetta Fabio Camilli and Diogo A. Gomes. Error estimates for the approximation of the effective Hamiltonian. Applied Mathematics and Optimization, 57(1):30-57, 2008.

[FM07] Albert Fathi and Ezequiel Maderna. Weak KAM theorem on non compact manifolds. NoDEA Nonlinear Differential Equations Appl., 14(1-2):1-27, 2007.

[FS04] A. Fathi and A. Siconolfi. Existence of $C^{1}$ critical subsolutions of the Hamilton-Jacobi equation. Invent. Math., 155(2):363-388, 2004.

[Gom08] D. Gomes. Generalized Mather problem and selection principles for viscosity solutions and Mather measures. To appear in Advances in Calculus of Variations, 2008.

[GV07] D. Gomes and E. Valdinoci. Generalized Mather problem and homogenization of Hamilton-Jacobi equations. in preparation, 2007.

[KRV06] Elena Kosygina, Fraydoun Rezakhanlou, and S. R. S. Varadhan. Stochastic homogenization of Hamilton-Jacobi-Bellman equations. Comm. Pure Appl. Math., 59(10):1489-1521, 2006.

[KV08] Elena Kosygina and S. R. S. Varadhan. Homogenization of HamiltonJacobi-Bellman equations with respect to time-space shifts in a stationary ergodic medium. Comm. Pure Appl. Math., 61(6):816-847, 2008.

[LS03] Pierre-Louis Lions and Panagiotis E. Souganidis. Correctors for the homogenization of Hamilton-Jacobi equations in the stationary ergodic setting. Comm. Pure Appl. Math., 56(10):1501-1524, 2003.

[LS05a] Pierre-Louis Lions and Panagiotis E. Souganidis. Homogenization of degenerate second-order PDE in periodic and almost periodic environments and applications. Ann. Inst. H. Poincaré Anal. Non Linéaire, 22(5):667$677,2005$. 
[LS05b] Pierre-Louis Lions and Panagiotis E. Souganidis. Homogenization of "viscous" Hamilton-Jacobi equations in stationary ergodic media. Comm. Partial Differential Equations, 30(1-3):335-375, 2005.

[Mad06] E. Maderna. On weak kam theory for $n$-body problems. preprint, 2006.

[Mat91] J. N. Mather. Action minimizing invariant measures for positive definite Lagrangian systems. Math. Z., 207(2):169-207, 1991.

[Mn96] Ricardo Mañé. Generic properties and problems of minimizing measures of Lagrangian systems. Nonlinearity, 9(2):273-310, 1996.

[Sou06] Panagiotis E. Souganidis. Recent developments in the theory of homogenization for fully nonlinear first- and second-order PDE in random environments. Bol. Soc. Esp. Mat. Apl. S $\overrightarrow{\mathrm{e} M A,(34): 81-90,2006 .}$

[Var08] S. R. Srinivasa Varadhan. Homogenization of random Hamilton-JacobiBellman equations. In Probability, geometry and integrable systems, volume 55 of Math. Sci. Res. Inst. Publ., pages 397-403. Cambridge Univ. Press, Cambridge, 2008.

[Vil03] Cédric Villani. Topics in optimal transportation, volume 58 of Graduate Studies in Mathematics. American Mathematical Society, Providence, RI, 2003.

São Paulo J.Math.Sci. 6, 2 (2012), 301334 